\title{
Foxq2 determines blue cone identity in zebrafish
}

2

3 Yohey Ogawa ${ }^{1, \dagger}$, Tomoya Shiraki $^{1, \dagger}$, Yoshitaka Fukada ${ }^{1, *}$ and Daisuke Kojima ${ }^{1, *}$ 4

$5 \quad{ }^{1}$ Department of Biological Sciences, School of Science,

6 The University of Tokyo, Hongo 7-3-1, Bunkyo-ku, Tokyo 113-0033,

7 Japan.

8

$9 \quad{ }^{*}$ Corresponding author. Email: sfukada@mail.ecc.u-tokyo.ac.jp (Y.F.);

10 sdkojima@mail.ecc.u-tokyo.ac.jp (D.K.)

$11+$ These authors contributed equally to this work

12

13

14

15

16

17 


\section{Abstract}

Most vertebrate lineages retain a tetrachromatic visual system, which is

4 supported by a functional combination of spectrally distinct multiple cone

5 photoreceptors, UV, blue, green, and red cones. The blue cone identity is

6 ensured by selective expression of blue (sws2) opsin, and the mechanism is

7 poorly understood because SWS2 gene has been lost in mammalian species

8 such as mouse, whose visual system has been extensively studied. Here we

9 pursued loss-of-function studies on transcription factors expressed

10 predominantly in zebrafish cone photoreceptors and identified Foxq2 as a core

11 factor driving sws 2 gene expression. foxq2 is expressed only in the blue cone,

12 and loss of foxq2 selectively abolishes sws 2 expression. Comparative genomic

13 analysis revealed that a wide range of vertebrate species retain both FOXQ2

14 and SWS2 genes. We propose that FOXQ2-dependent SWS2 expression is a

15 prevalent regulatory mechanism that was acquired at the early stage of

16 vertebrate evolution 


\section{Introduction}

Most vertebrates have highly developed camera-type eyes with duplex retinas equipped with rod and cone photoreceptor cells (1-3). Rods with a

4 higher light-sensitivity respond to single photons and mediate scotopic vision under twilight conditions at night. In contrast, cones show a relatively lower sensitivity without saturating in brighter light and mediate photopic vision under a daylight condition. Color discrimination is established by a combination of spectrally distinct cone subtypes, each expressing a single cone opsin out of four subfamilies: UV- (SWS1, wavelength of maximum sensitivity [ $\left.\lambda_{\max }\right]$ : $360-$ $420 \mathrm{~nm}$ ), blue- (SWS2, $\left.\lambda_{\max }: 400-470 \mathrm{~nm}\right)$, green- (RH2, $\left.\lambda_{\max }: 460-510 \mathrm{~nm}\right)$ and red-sensitive opsins (LWS, $\left.\lambda_{\max }: 510-560 \mathrm{~nm}\right)(4,5)$. Most vertebrates retain the tetrachromatic visual system organized by the four cone opsin subfamilies. A full set of genes encoding the four cone opsins are present in the southern hemisphere lamprey, a jawless vertebrate belonging to the earliest-branching vertebrate group (6). This fact supports the idea that the last common ancestor of vertebrates should have possessed color vision based on the four cone opsin subfamilies $(4,7)$.

Retinal progenitor cells differentiate into all types of retinal neurons in a temporal order, conserved among many species (8-10). In the later process, transcription factors regulate photoreceptor-specific gene expression. Cone-rod homeobox $(\mathrm{Crx})$ is an upstream transcriptional regulator for both rod and cone photoreceptors $(11,12)$. A rod master regulator, neural retina leucine zipper $(\mathrm{Nrl})$, and its downstream factor, Nuclear Receptor 2E3 (Nr2e3), enhance rodspecific gene expression and repress SWS1 expression $(13,14)$. With regard to cone subtypes, thyroid hormone receptor beta (Thrb) is a master transcriptional regulator for expression of LWS opsin and responsible for differential expression between LWS and SWS1 opsins in mice (15), zebrafish (16), and human (17). Another transcription factor, T-box 2b (Tbx2b), plays an essential role in SWS1 opsin expression in zebrafish (18). On the other hand, much less is known about a regulatory network governing expression of the middle wavelength-sensitive opsin genes, sws2 and rh2, which have been lost in most mammalian species. 
In zebrafish, a tetrachromatic freshwater fish, we found that sine oculis

2 homeobox 7 (Six7) is required for expression of all the four subclasses of $r h 2$

3 genes (rh2-1, rh2-2, rh2-3 and $r h 2-4)(19,20)$, which are tandemly arrayed,

4 expressed in different cone cells, and spectrally distinct from each other (21,

5 22). Six7 and its homolog Six6b control sws2 expression as well (23). The

6 cone-enriched transcription factors, Six7 and Six6b, share common DNA-

7 binding sites in both $r h 2$ and sws 2 gene loci, and Six $6 \mathrm{~b}$ overexpression rescues

8 the reduced level of $r h 2$ expression in six7 deficient fish (23). The overlapping

9 functions between Six6b and Six7 for sws2 and rh2 expression imply that an

10 additional factor(s) directs selective expression of either sws2 or rh2 gene and

11 determines the identities of the middle wavelength-sensitive cone subtypes. In

12 the present study, gene expression profiling with isolated rods and cones

13 enabled us to identify a list of cone-enriched transcription factors. Our in vivo

14 functional analyses revealed a core transcriptional network, in which Foxq2 acts

15 as a downstream regulator of Six 7 and regulates sws 2 expression. We

16 demonstrate that Foxq2 is a terminal selector determining SWS2 cone identity

17 during development of the middle wavelength-sensitive cone subtypes. 


\section{Results}

\section{A severe reduction of sws2 expression in foxq2 mutant \\ 3 zebrafish}

Six6b and Six7 are predominantly expressed in zebrafish cone photoreceptors and responsible for expression of the middle wavelengthsensitive opsin genes, sws2 (blue) and rh2 (green) (23). To identify a transcription factor(s) that governs differentiation between SWS2 and RH2 cone subtypes, we searched for cone-specific genes by comparing gene expression profiles between cones and rods. These photoreceptor cells were purified from the retinas of transgenic adult zebrafish, each of which express EGFP in all cone subtypes [Tg(gnat2:egfp)] or in rods [Tg(rho:egfp)] (19). The cone- or rodenrichment in these purified samples was validated by RT-qPCR analyses of cone- and rod-specific transducin alpha-subunit genes, i.e., gnat2 and gnat1, respectively (Fig. 1A). A subsequent microarray analysis revealed approximately 500 genes showing more than 10-fold higher expression in cones than in rods (Data S1). These cone-enriched genes included four transcription factors, foxq2, E2F transcription factor 7 (e2f7), nuclear factor 1A (nfia), and nuclear receptor $2 \mathrm{~F} 6 \mathrm{~b}$ ( $n r 2 f 6 b)$, the roles of which in photoreceptor development were not known. Genomic loci of these genes harbor Six6b- and Six7-binding sites as revealed by our ChIP-seq analysis (23) (Fig. S1), implying that some of these cone-enriched transcription factors mediate a regulatory function(s) downstream of Six6b and Six7. In addition, we paid attention to two coneenriched transcription factors, $t b x 2 b$ and thrb. They are known to be required for expression of sws1 (18) and IWs (16), respectively, but their contributions to the sws2 and rh2 gene expression have not been well characterized. Coneenriched expression of these six transcription factors was verified by RT-qPCR analyses with purified cone and rod cells (Fig. 1A).

We generated loss-of-function mutants of zebrafish for each of the six transcription factors by introducing a frameshift mutation. In these mutants, ocular transcript levels of the middle wavelength-sensitive opsin genes were examined by RT-qPCR analysis (Fig. 1B). Among the mutant larvae, a foxq2 mutant (Fig. 1C, ja74) displayed the most striking reduction in sws2 expression 
1 as compared to the WT siblings (Fig. 1B). Another mutant line of foxq2 (Fig. 1C, 2 ja77) similarly showed a severe reduction of sws2 expression in the larvae (Fig. 3 S2A). The decrease of sws2 expression in the mutant larvae (Fig. 1D and S2A) was not due to delayed development of SWS2 cones because the adult foxq2 mutant (ja77) also exhibited minimal expression of sws2 gene (Fig. S2B). In the foxq2 mutant retinas, in situ hybridization signals of sws2 transcripts were undetectable with no apparent change in retinal morphology at the larval (Fig. 1E) and adult stages (Fig. S2C). These results demonstrate that foxq2 is indispensable for sws2 expression in SWS2 cone subtype. In parallel, the foxq2 mutation caused significant increase in mRNA levels of two major rh2 subclasses, rh2-1 in the larvae (Fig. S2A, ja77) and rh2-2 in the adult (Fig. S2B, ja77), as well as upregulation of $r h 2-2$ in the larvae (Fig. 1D, ja74 and Fig. S2A, ja77) and $r h 2-4$ in the adult (Fig. S2B, ja77). The upregulation of $r h 2$ gene expression is likely due to transcriptional compensation for severe reduction of sws 2 expression caused by the loss of Foxq2 function.

$\mathrm{Tbx} 2 \mathrm{~b}$ is also a cone-specific transcription factor (Fig. 1A) and is required for expression of sws 1 gene (18). In the present study, $t b \times 2 b^{j a 20}$ mutant was designed to encode C-terminally truncated Tbx2b protein (Fig. 1F), which lacked two DNA-recognition helices of T-box domain (24) in a manner similar to $t b \times 2 b^{f b y}$ mutant (25). As reported in $t b \times 2 b^{f b y}(18), t b \times 2 b^{j a 20}$ larvae exhibited a severe decrease of sws 1 and a parallel increase of rhodopsin (rho) expression (Fig. 1F and 1G). Of note, we noticed that $t b \times 2 b^{j a 20}$ mutation caused $40 \%$ reduction of sws2 expression level from that in the WT siblings and substantial increase in mRNA levels of $r h 2-1$ and $r h 2-2$ (Fig. 1G). Although the effect of $t b x 2 b$ mutation on sws 2 expression was weaker than that of foxq 2 mutation, $t b x 2 b$ appears to play a supportive role to foxq 2 on sws 2 expression in addition to the essential role on sws1 expression.

Thrb is another cone-specific transcription factor (Fig. 1A), and its mutant $29 t_{h r b}^{j a 27}($ Fig. 1H) manifested a massive reduction of both Iws1 and Iws2 30 expression and a concomitant increase in sws1 expression in the larval eyes 31 (Fig. 1I) as reported in previous studies (16, 26-28). We noticed that thrbia27 32 mutant exhibited a moderate but significant increase in sws2 gene expression 33 (Fig. 1I). The sws2 upregulation accompanied no significant reduction of $r h 2$ 
1 expression level (Fig. 1I). Accordingly, Thrb contributes to fine-tuning of sws2

2 expression together with a dominant role in regulation of Iws expression. In

3 contrast, nfia, nr2f6b, and e2f7 mutant fish showed no noticeable change in sws2 or rh2 expression (Fig. 1B and S3). These three transcription factors are dispensable for middle-wavelength cone opsin gene expression.

Collectively, our mutant analysis demonstrates that sws 2 expression is predominantly regulated by a cone-specific transcription factor, Foxq2, with the aid of regulation by Tbx $2 b$ and Thrb.

\section{SWS2 cone subtype-specific expression of foxq2}

To gain insights into how foxq2, tbx $2 b$, and thrb regulate sws2 and $r h 2$ expression, we investigated gene expression profiles of foxq2, $t b \times 2 b$, and thrb among the four cone subtypes: SWS1, SWS2, RH2, and LWS. These four cone cells were isolated from four different lines of transgenic fish, each of which expresses EGFP in one of the four cone subtypes (Fig. 2A; see Materials and Methods for details). The cone subtype-enrichment in these purified samples was validated by RT-qPCR analyses of cone opsin genes (Fig. 2B). Subsequent analysis of foxq2, $t b \times 2 b$, thrb, six $6 b$, and six 7 in the four samples revealed that foxq2 was specifically expressed in SWS2 cone subtype (Fig. 2C). Another SWS2 regulator, $t b x 2 b$, was expressed in both SWS1 and SWS2 cone subtypes, whereas thrb was expressed only in the LWS cone subtype (Fig. 2C). In contrast, SWS2/RH2 regulators, six $6 b$ and six7, were expressed in all the cone subtypes (Fig. 2C). The SWS2 cone subtype-enriched expression of foxq2 and $t b \times 2 b$ suggests that these two factors coordinately regulate the cell-typespecific expression of sws 2 .

foxq2 expression was restricted to the retina among various adult tissues (Fig. 2D), and in the larvae, it was detected only in the anterior half of the body (including the eyes) but not in the posterior half (Fig. 2E). These observations suggest that in the course of development, foxq2 expression becomes restricted to SWS2 cones within the retina. Importantly, the mRNA level of foxq2 was severely reduced in six6a/six6b/six7 triple knockout (TKO) lacking sws2 expression, i.e., loss of SWS2 cone identity from the retina (23) (Fig. 2F and G). 
1 foxq2 expression was also abrogated in the larval eyes of foxq2 mutant (ja74)

2 as compared to that of WT sibling (Fig. 2H). The close correlation between

3 foxq2 and sws2 expressions imply that Foxq2 is responsible for establishing

4 SWS2 cone identity. Meanwhile, $t b x 2 b$ was expressed widely in various tissues

5 at the larval (Fig. 2E) and adult stage (Fig. 2D), and the ocular $t b x 2 b$ expression

6 level was unaffected in the foxq2 mutant (Fig. 2H) and six6a/six6b/six7 TKO

7 (Fig. 2F and 2G), both of which were deficient in sws2 expression. These

8 observations indicate dominant expression of $t b x 2 b$ outside of SWS2 cones and

9 imply that Tbx2b may have pleiotropic roles for eye development (29) including

10 the cone identity determination.

\section{Functional interaction of Foxq2 with sws2 promoter}

We then investigated functional interaction of Foxq2 with sws2 promoter. Foxq2 protein (Fig. 1C) has a conserved DNA-binding domain, termed forkhead domain composed of about 100 amino acid residues (30). In Fox transcription factor family, Foxq2 is categorized into clade I forkhead proteins (31), which recognize two types of common forkhead-target DNA motifs termed FkhP (RYAAAYA) and FkhS (AHAACA) (32). Our motif scanning analysis (see Materials and Methods) revealed two FkhP motifs and two FkhS motifs present within 1.56-kb sws2 promoter region (Fig. 3A and S4), which drives selective gene expression in SWS2 cones (33). In a cell-based reporter assay, the 1.56kb sws2 promoter was transactivated by VP64-Foxq2 (Fig. 3B and 3C), in which Foxq2 is N-terminally fused with four repeats of the VP16 transcriptional activator domain [Fig. 3B and (34)]. The transcriptional activation was attenuated by deletion of the upstream region of 1.03 or $1.26 \mathrm{kbp}(0.53-\mathrm{kb}$ or 0.3-kb sws2 promoter, respectively), leaving single FkhS motif (Fig. 3A and 3C). Still, we observed more than 30-fold transactivation of the $0.3-\mathrm{kb}$ sws2 promoter by VP64-Foxq2, while the activation was largely reduced by the complete deletion of the promoter sequence (Fig. 3C). The VP64-Foxq2-dependent transactivation of a shorter promoter (0.25-kb sws 2 promoter) was markedly decreased by introducing a 6-bp mutation in the FkhS motif (Fig. 3D). We then 
1 that are required for specific DNA-binding of the forkhead domain (32) (Fig. 3B).

2 The protein mutation abolished the ability of the transcriptional activation of the 3 sws2 promoter (Fig. 3B, 3D and 3E). These results demonstrated that Foxq2

4 functionally interacted with the forkhead-target DNA motif in the sws2 promoter.

5 Together with the SWS2-specific expression of foxq2 (Fig. 2), Foxq2-mediated

6 transcriptional regulation of sws 2 gene would reasonably account for the

7 selective expression of sws2 opsin in SWS2 cone subtype.

\section{$9 \quad$ Fate determination of SWS2 cone subtype governed by foxq2}

The foxq2 mutants (ja74 and ja77) were not only deficient in sws2 expression but also characterized by significant reduction in mRNA level of arrestin $3 b(a r r 3 b)$, a cone phototransduction gene selectively expressed in SWS1 and SWS2 cone subtypes (35) (Fig. 1D and S2). These observations suggest that foxq2 deficiency impairs development of SWS2 cone cells and/or their maintenance. To gain further insight into the fate determination of SWS2 cone subtype, we generated foxq2 transgenic (foxq2-tg) zebrafish, $\mathrm{Tg}$ (5.2crx:EGFP-2A-FLAG-foxq2), in which expressions of both EGFP and Foxq2 are driven by 5.2-kb crx promoter in all the developing and matured photoreceptor cells (Fig. 4A and 4B) (16). RT-qPCR analysis of larval ocular mRNAs in three foxq2-tg lines (ja78Tg, ja79Tg, and ja91Tg) revealed that forced expression of foxq2 in the wild-type background caused a severe decrease in sws1 mRNA level (Fig. 4C and S5). This phenotype was not accompanied by any detectable change in the expression levels of $t b x 2 b$ and crx (Fig. 4D and S5), which are regulators of sws1 expression (18) (Fig. 1G). It is most probable that Foxq2 suppresses sws1 expression in cones (Fig. 4E).

The selective expression of foxq2 in SWS2 cones (Fig. 2C), together with 27 binding of Six6b and Six7 to foxq2 gene locus (Ref. 23 and Fig. S1), suggest 28 that sws2 expression is regulated by Foxq2 downstream of Six6b and Six7 (Fig. $4 \mathrm{E})$. This possibility was explored by overexpressing foxq 2 in the homozygous six7 knockout (KO) (19), in which expression levels of sws2 and foxq2 are markedly reduced (Fig. 4C and 4D). The forced expression of foxq2 in the six 7 $\mathrm{KO}$ background (foxq2-tg;six7 KO) recovered the reduced expression of sws2 
1 up to a level higher than that in the WT control (Fig. 4C). In contrast, the

2 overexpression of foxq2 had no significant effect on the severely reduced

3 expression levels of $r h 2$ (rh2-1 plus rh2-2) (Fig. 4C) or on mRNA levels of $c r x$,

$4 t b x 2 b$, six $6 b$, and $\operatorname{six} 7$, transcriptional regulators of cone opsin expression (Fig.

5 4D). These results demonstrated that Foxq2 regulates sws2 expression

6 downstream of Six7, suggesting that Foxq2 is a terminal selector for SWS2

7 subtype identity (Fig. 4E).

8

\section{Conservation of FOXQ2 gene among vertebrate species}

FOXQ2 gene is annotated in the genomes of a wide range of vertebrate species (Fig. 5), such as ray-finned fish (spotted gar, zebrafish, and medaka), a lobe-finned fish (coelacanth), and an avian (sparrowhawk), all of which retain SWS2 gene. We found highly conserved gene synteny around FOXQ2 locus among the vertebrates, and the synteny analysis of the human genome indicated the absence of any gene homologous to FOXQ2 between PIAS4 and ZBTB7A gene loci (Fig. 5B). Intriguingly, BLAST searching and subsequent manual annotation revealed that a mammalian species, platypus, retains a gene orthologous to FOXQ2 harboring the forkhead domain highly conserved among FOXQ2 subfamily members (Fig. 5 and S6; see also supplementary text). The oviparous mammals including platypus diverged from marsupial and placental mammals at the earlier stage of mammalian evolution (around 200 million years ago). Of note, platypus retains SWS2 gene in its genome (36), whereas many other mammals have lost it $(7,37)$ most likely due to a long evolutionary history of nocturnality in mammalian ancestors (38). These lines of genomic evidence suggest that Foxq2-dependent SWS2 expression is a highly conserved regulatory mechanism that was acquired at the early stage of vertebrate 27 evolution. 


\section{Discussion} on the earth's surface are enriched with blue-to-green region of visible spectrum (39), which is detected by cone photoreceptor cells expressing middlewavelength sensitive opsin genes, SWS2 and $\mathrm{RH} 2$. Their expression requires transcription factors, Six6 and Six7, in zebrafish $(19,23)$, and the six6/six 7 mutant fish lacking both SWS2 and RH2 cones show severely reduced survival rate due to impairment of visually driven foraging behavior (23). A recent study of in vivo calcium imaging of zebrafish cone photoreceptors reported that SWS2 and RH2 cones, but not SWS1 or LWS cones, display strong spectral opponency and efficiently extract chromatic information from the natural light spectrum (40). In this way, a combination of spectrally distinctive SWS2 and $\mathrm{RH} 2$ cones particularly plays an important role in the tetrachromatic visual system. The present study explored the transcriptional regulatory logic that defines SWS2 and RH2 cone identities. For this purpose, cone-enriched transcription factors were comprehensively identified by the transcriptome analysis with purified cone cells (vs. purified rod cells; Data S1). Subsequent functional analyses of these transcription factors demonstrated that Foxq2 is indispensable for sws2 expression (Fig. 1). We pursued expression profiling of the cone-enriched transcription factors among the four isolated cone subtypes (Fig. 2A-C). foxq2 is selectively expressed in SWS2 cone, whereas all cone subtypes express six $6 b$ and six 7 (Fig. 2C), which are required for both sws2 and $r h 2$ gene expression (19). A transcriptional network is deciphered in which Foxq2 acts as a downstream regulator of Six7 (Fig. 4A-D) and regulates sws2 expression (Fig. 3). A wide range of vertebrate species retain both FOXQ2 and SWS2 gene (Fig. 5). These lines of evidence demonstrate that Foxq2 determines SWS2 cone identity in zebrafish (Fig. 4E) and suggest that Foxq2dependent sws2 expression is a highly conserved regulatory mechanism that was acquired at the early stage of vertebrate evolution.

Quantitative comparison of transcription factor expression among the four isolated cone subtypes (Fig. $2 \mathrm{C}$ ) provides valuable information about terminal differentiation of each cone subtype in zebrafish. foxq2, a crucial regulator of sws 2 expression, is selectively expressed in SWS2 cones, indicating that Foxq2 
1 is a bona fide terminal selector of SWS2 cone. On the other hand, $t b x 2 b$, known

2 as a master regulator of SWS1 cone (18), is expressed in not only SWS1

3 cones, but also SWS2 cones (Fig. 2C), in which Tbx2b acts as a supportive

4 regulator of sws2 expression (Fig. 1G). The expression of the SWS1 master

regulator in SWS2 cones implies that some mechanism should suppress sws1 misexpression in SWS2 cones. It is probable that Foxq2 contributes to the sws1 suppression because the forced expression of foxq2 markedly reduced sws 1 expression in SWS1 cones (Fig. 4C and S5). Similarly, Foxq2 appears to suppress rh2 misexpression in SWS2 cones (Fig. 1D, 4C, and S2). Thus, Foxq2 has dual functions acting as an activator of sws 2 transcription and as a suppressor of sws1 and rh2 genes in SWS2 cones (Fig. 4E) and, presumably, in developing cone cells. The dual functions of Foxq2 would enhance robustness of SWS2 cone identity. Meanwhile, thrb is predominantly expressed in LWS cone subtype (Fig. 2C), being consistent with the widely accepted idea that Thrb is a master regulator for the LWS cone (15-17). Collectively, Foxq2, Tbx2b, and Thrb can be defined as the terminal selectors (41) that govern cell fate determination of SWS2, SWS1, and LWS cone subtypes, respectively.

In contrast to these three terminal transcriptional selectors, six 7 is widely expressed among the four cone subtypes (Fig. 2C) and thus six 7 is unlikely to be a terminal selector of $\mathrm{RH} 2$. Although Six7 is indispensable for expression of rh2 (rh2-1, rh2-2, rh2-3, and rh2-4; Ref. 19 and Fig. 4C), terminal differentiation of $\mathrm{RH} 2$ cone might be mediated by an unidentified transcription factor. An alternative idea is that Rh2 cone identity is established by both (i) the presence of six7 and (ii) the absence of the terminal selectors such as tbx $2 b$ (for SWS1), foxq2 (for SWS2), and thrb (for LWS). In this scenario, differentiation toward Rh2 cones should be a default pathway in the cone development, because Rh2 cone differentiation is governed by $\operatorname{six} 7(19)$ and $\operatorname{six} 7$ expression begins as early as that of $c r x$, a master regulator of cone and rod development $(19,39)$. It is worth noting that the "Rh2-default" hypothesis is consistent with molecular phylogeny of cone opsin genes, in which $\mathrm{RH} 2$ subfamily diverged from SWS2 at the latest step of the molecular evolution of the cone opsins $(4,5,40)$. Building on these assumptions, we propose that the tetrachromatic color vision system in ancestral vertebrates employed the "RH2-default" mechanism of cone 
1 differentiation as is observed in zebrafish. In mammals, on the other hand, $\mathrm{RH} 2$

2 and SWS2 genes have been lost, and hence it is likely that the "RH2-default"

3 mechanism has been modified to a "SWS1-default" mechanism. In the latter

4 mechanism, the presence or absence of only one terminal selector, THRB,

5 directs differentiation between LWS and SWS1 cones as shown in mice $(8,15)$.

FOXQ2 gene is evolutionarily conserved not only among vertebrates but also in many invertebrates $(31,32,44)$. In embryos of the invertebrates, FOXQ2 is responsible for specification and positioning of anterior neuroectoderm (45,

9 46). The anterior neuroectoderm develops into central nervous system, in which

10 a subset of sensory cells express a photoreceptive protein, c-opsin, that is

11 orthologous to vertebrate cone and rod opsins $(47,48)$. FOXQ2 might regulate

12 differentiation of the c-opsin-expressing cells from progenitor cells in the

13 anterior neuroectoderm. Intriguingly, a transcriptional network in the anterior

14 neuroectoderm of invertebrates also includes six3, a gene orthologous to

15 vertebrate $\operatorname{six} 3, \operatorname{six} 6$ and $\operatorname{six} 7(45,46)$. The presence of the Six3/6/7-Foxq2

16 transcriptional network in both vertebrate and invertebrate species suggest that

17 their common ancestor employed this transcriptional network for development

18 of the light-sensitive cells. In the vertebrate lineage, the Six3/6/7-Foxq2

19 transcriptional network might have been co-opted for establishment of SWS2

20 cone identity during or after the appearance of a full set of cone opsins, thereby

21 conferring high-acuity discrimination of blue-to-green region of visible light. 


\section{Materials and Methods}

3 Zebrafish. The Ekkwill strain was used as the wild-type zebrafish. All

4 appropriate ethical approval and licenses were obtained from Institutional

5 Animal Care and Use Committees of The University of Tokyo. All the

6 procedures were conducted according to local guidelines of the University of

7 Tokyo. Zebrafish were raised in a 14-h light/10-h dark cycle and fed twice per

8 day with live baby brine shrimps. Embryos were raised at $28.5{ }^{\circ} \mathrm{C}$ in egg water

9 (artificial seawater diluted 1.5:1,000 in water). Mutant strains and transgenic

10 lines used in the present study are listed in Table S1.

12 Purification of rod and cone photoreceptor cells. For isolation of rod and

13 cone photoreceptor cells, we used the transgenic zebrafish lines, $T g(\text { rho:egfp })^{j a 2}$

14 (49) and $T g$ (gnat2:egfpjia23 (19), which express EGFP in rods and all cone

15 subtypes, respectively. The rod and cone cells were isolated as described

16 previously $(19,23,50)$. Briefly, retinas were dissected from dark-adapted adult

17 fish under dim red light. The isolated retinas were digested with $0.25 \%$ trypsin,

$1810 \mathrm{U} / \mathrm{ml}$ DNasel, 2 mM MgCl 2 , and 2 mM EGTA in $\mathrm{Ca}^{2+}$-free Ringer's solution

19 for $30 \mathrm{~min}$ at $37^{\circ} \mathrm{C}$. The reaction was terminated by adding soybean trypsin

20 inhibitor (final 0.5\%) and fetal bovine serum (final 10\%). The dissociated cells

21 were filtrated through a 35- $\mu$ m nylon mesh (Falcon). EGFP-positive cells were

22 isolated with a fluorescence activating cell sorter (FACSAria, BD Biosciences)

23 by the following three parameters: forward scatter, side scatter and green

24 fluorescence. The isolated cells were directly collected into $800 \mu \mathrm{L}$ of TRIzol

25 reagent (Thermo Fisher Scientific) in $1.5 \mathrm{ml}$ microtubes.

26 For isolation of cone subtypes, we used four lines of transgenic

27 zebrafish: $\operatorname{Tg}(-5.5 o p n 1 s w 1: E G F P)^{k j 9}(51), \operatorname{Tg}(-3.5 o p n 1 s w 2: E G F P)^{k j 11}$ (33),

$28 T g(R H 2-2 / G F P-P A C)^{k j 4}(52)$ and $T g(-0.6 o p n 1 / w 1-/ w s 2: G F P)^{k j 19}$ (53), which

29 express EGFP in SWS1, SWS2, RH2 and LWS cone subtypes, respectively.

30 The cone photoreceptor isolation was carried out as described above with some 31 modifications as follows. The isolated cells were directly collected into $450 \mu \mathrm{L}$ of

32 the lysis buffer (RNeasy Lysis Buffer, Qiagen) in $1.5 \mathrm{ml}$ microtubes. 


\section{Microarray.}

Total RNA was isolated from the sorted cells using RNeasy Extraction kit (Qiagen). Quality and quantity of the resulting RNA were assessed using a NanoDrop ND-2000 spectrophotometer (Thermo Scientific) and an Agilent 2100 Bioanalyzer (Agilent Technologies). Microarray Analysis was performed using the Agilent $4 \times 44 \mathrm{k}$ Zebrafish microarray according to the manufacturer's protocol for the two-color method. Cy3- or Cy5- labeled cRNA probe was synthesized from $150 \mathrm{ng}$ of total RNA using the Quick-Amp Labeling Kit (Agilent Technologies). Quantity of the resulting labeled cRNA was assessed using a NanoDrop ND-2000 spectrophotometer. Equal amounts of Cy3 and Cy5-labeled cRNA (825 ng) from two different samples were hybridized to zebrafish microarrays (Agilent Zebrafish Oligo Microarrays ver.2, G2519F-019161) for 17 $\mathrm{h}$ at $60^{\circ} \mathrm{C}$. In order to compare gene expression profiles between cone and rod samples, we analyzed two independent biological replicates as following: (1) Cy3-rod\#1 cRNA and Cy5-cone\#1 cRNA and (2) Cy5-rod\#2 cRNA and Cy3cone\#2 cRNA. The hybridized arrays were then washed and scanned using an Agilent microarray scanner (G2505 C; Agilent Technologies). Data were extracted from the scanned image using Feature Extraction version 10.5.1.1 (Agilent Technologies). We then listed differentially expressed genes with Microsoft Excel as follows: 1) We excluded any probes whose signals in two arrays were all determined as negative, meaning that probes were not flagged as "WellAboveBG" in any of two arrays. 2) We selected all the probes whose signal intensities vary largely between the photoreceptor cell types according to the following thresholds of the averaged ratios: 10-fold increase for cone compared with rod and 4-fold increase for rod compared with cone. 3) We checked whether both of the two independent biological replicates showed similar expression profiles, and selected probes whose absolute values of the ratios in the two biological replicates were both $>2.0$ for $\mathrm{rod} /$ cone or both $>3.0$ for cone/rod. The lower threshold was given for the rod/cone ratio because a small but noticeable level of rod contamination was detected in cone samples such as gnat1 (Fig.1A). We set these threshold values based on the ratios observed for known cone-specific genes. The microarray datasets will be 
1 available at the National Center for Biotechnology Information Gene Expression

2 Omnibus (GEO) database.

3

4 Generation of mutant zebrafish. The foxq2, thrb, and $n r 2 f 6 b$ mutants were

5 generated with a CRISPR/Cas9 system. The sgRNA sequences (Table S2)

6 were designed to target exon 1 or exon 2 (Fig. 1C, 1H, and S3E) using

7 CRISPRdirect (https://crispr.dbcls.jp/) (54), CHOPCHOP

8 (https://chopchop.cbu.uib.no) (55), or CRISPRscan (56). The sgRNA was

9 synthesized by a cloning-free method as previously described (57). For

10 generating Cas9 mRNA, the template plasmid DNA, pCS2-nCas9n (addgene

11 \#47929) (58) or pCS2+hSpCas9 (addgene \#51815) (59) were used for in vitro

12 transcription. Cas9 mRNA was synthesized with the SP6 mMESSAGE

13 mMACHINE Kit (Ambion) and purified with the RNeasy Mini kit (Qiagen). The

14 solution containing 200-pg Cas9 mRNA and 25-pg sgRNA was injected into the

15 cytoplasm of the one cell-stage embryos.

16 The $t b \times 2 b, e 2 f 7$, and nfia mutants were generated by TALENs as

17 previously described $(19,23)$. To target each of the three genes, a pair of the

18 TAL effector repeats (Table S2) were designed to target exon 2 or exon 3 with

19 the Golden Gate assembly methods (Fig. 1, S3A, and S3C). TALEN mRNA was

20 synthesized using the SP6 mMESSAGE mMACHINE Kit (Ambion) and purified

21 with the RNeasy Mini kit (Qiagen). The solution containing 200-pg each of the

22 two TALEN mRNAs was injected into the cytoplasm of the one cell-stage

23 embryos.

The injected fish (F0) were crossed with the wild-type zebrafish. The resultant F1 offspring were screened for the presence of CRISPR/Cas9- or TALENs-induced mutations by a combination of PCR and subsequent enzyme digestion for thrb, $n r 2 f 6 b, t b x 2 b$, e2f7, and nfia mutants, or by Heteroduplex Mobility Assay (HMA) for foxq2 mutants as described previously (60). To sequence-verify mutations, genomic sequences surrounding the mutations were amplified by nested PCR, and the resultant PCR products were sequenced directly. After isolation of mutant zebrafish and verification of the mutations, the mutant genotypes were confirmed by a combination of PCR and subsequent enzyme digestion except for the foxq2 (ja74) gene locus. The foxq2ja74 mutant 
1 genotype was determined by PCR with two pairs of primers: (i) foxq2_Fw1A (5'-

2 TGGCT AAACG AACAA ACACG -3') and foxq2_Rv1WT (5'- ATGGA TTGAC

3 ATTGT CCTCT G -3'); (ii) foxq2_Fw1mutA (5'- GCTGG AAGAG CAGAA

4 CAATG -3') and foxq2_Rv1A (5'- GGAAA TGAGG GCAAT GTAGG -3'). PCR

5 primers used for amplification are listed in Table S3.

The mutant larvae and its siblings were dissected into anterior and posterior segments; the posterior parts were used for genotyping, while the anterior segments were soaked in RNAlater (Sigma) for RT-qPCR analysis or fixed with 4\% paraformaldehyde (PFA) in $\mathrm{Ca}^{2+}$ - and $\mathrm{Mg}^{2+}$-free Dulbecco's PBS (D-PBS) for cryosectioning.

RT-qPCR analysis. Zebrafish were anesthetized by chilling on ice, and their tissues were collected during the light phase of the light-dark cycle and soaked in RNAlater. After genotyping described in the previous section, the larval eyes were isolated with a needle. A pair of two larval eyes or one adult eye was considered a biological replicate for each genotype. RNA extraction and reverse transcription were conducted as described previously $(19,23)$. In short, RNA was extracted and purified with RNeasy Micro Kit or RNeasy Mini Kit (Qiagen). In all the experiments except for Fig. 1A, the extracted RNA was reversetranscribed into cDNA with the oligo $(\mathrm{dT})_{15}$ primer with GoScript ${ }^{\mathrm{TM}}$ Reverse Transcriptase (Promega). In Fig. 1A, the reverse transcription was conducted with SuperScript II (Thermo Fisher Scientific) using anchored (dT) 16 primers. The reverse-transcribed cDNA was subjected to quantitative PCR using GoTaq qPCR Master Mix (Promega) and the StepOnePlus ${ }^{T M}$ Real-time PCR system (Applied Biosystems) following the manufacturers' protocols. Expression levels were calculated by the relative standard curve method. The standard curve was prepared with serial dilutions of cDNA samples reverse-transcribed from total RNA of zebrafish eye. The transcript levels were normalized to beta-actin 2 (actb2) transcript levels in all the figures. Primers used for quantitative PCR are listed in Table S4 and in our previous studies $(19,23)$. Total transcript level of $\mathrm{RH} 2$ (rh2-1 and rh2-2) or LWS (Iws1 and Iws2) opsin genes at the larval stage was measured with a set of PCR primer, which amplifies both $r h 2-1$ and $r h 2-2$ opsin genes (referred to here as rh2-1/2) or both Iws1 and Iws2 opsin genes 
1 (referred to here as Iws 1/2). Expression levels of all the transcript isoforms of

2 thrb were measured in Fig. 1A, while in the rest of the experiments, we

3 measured expression levels of a transcript isoform of thrb, thrb2, which is

4 essential for Iws expression in mouse and zebrafish $(15,16)$.

In situ hybridization. in situ hybridization using ocular sections was carried out as described previously $(19,23)$. In short, zebrafish were anesthetized by chilling on ice and subjected to dissection the light phase of the light-dark cycle. The larval anterior segments or the adult eyes were fixed in 4\% PFA in D-PBS overnight at $4{ }^{\circ} \mathrm{C}$. Before the fixation, the adult eyes were enucleated and poked with tweezers to make a tiny hole in the cornea. After sucrose infiltration and OCT compound embedding, the 10- $\mu \mathrm{m}$ frozen ocular sections were prepared with a cryostat. The cryosections were pre-treated with proteinase $\mathrm{K}$ and hybridized with DIG-labelled cRNA probes, and the hybridization signals were visualized by NBT/BCIP staining. The images were acquired with an upright microscope (Axioplan2, Carl Zeiss). The cRNA probes were generated as described in our previous study $(19,23)$.

Immunohistochemistry. Immunohistochemistry with ocular sections was carried out as described previously $(19,23)$. Briefly, ocular cryosections were prepared as described in the previous section. The cryosections were pretreated with a blocking solution and then incubated with a primary antibody diluted in the blocking solution overnight at $4^{\circ} \mathrm{C}$. After washed with PBS $(10 \mathrm{mM}$ Na-phosphate buffer, $140 \mathrm{mM} \mathrm{NaCl}, 1 \mathrm{mM} \mathrm{MgCl}_{2}, \mathrm{pH} 7.4$ ), the treated sections were immersed again with the blocking solution, and then incubated for $4 \mathrm{hr}$ at room temperature with a secondary antibody and with DAPI $(3 \mu \mathrm{g} / \mathrm{ml})$ for staining of the cell nuclei. The stained sections were coverslipped with VECTASHIELD Mounting Medium (Vector Laboratories) and imaged with a confocal laser scanning microscope (TCS SP8, Leica). The primary antibody used is mouse monoclonal antibody Zpr1 (diluted 1:400, Zebrafish International

31 Resource Center, Eugene) against arrestin 3a. The secondary antibody used is goat anti-mouse IgG antibody conjugated with Alexa-568 (diluted to $2 \mu \mathrm{g} / \mathrm{ml}$, A11004, Molecular Probes). 
2 Luciferase assay. For constructing the firefly luciferase reporter plasmids, the

3 1.56-kb sws2 upstream region with its 5'-UTR was amplified by PCR. The

4 amplified fragment was ligated using the In-Fusion cloning kit (Takara) into the pGL4.13[luc2/SV40] (E6681, Promega) digested with HindIII and Bg/lI. PCR primers used were as follows: IF-sws2-1.5kFwBgl (5'- CGAGG ATATC AGATC TAACG ATGTT TGCTG TTTGT TC -3') and IF-sws2-RvHind (5'- CCGGA TTGCC AAGCT TCTTG CTTGT AATTG GTGCC C -3'). The 0.53-kb sws2 reporter was constructed in a manner similar to the 1.56-kb sws2 reporter with following primers: IF_532_sw2_FwXho (5'- GCTCG CTAGC CTCGA GCAAC TCTCA AGTAT TTAAG G -3') and IF-sws2-RvHind. The 1.56-kp sws2 reporter was truncated by PCR to generate the $0.3-\mathrm{kb}$ and $0.25-\mathrm{kb}$ sws 2 reporter by using the following PCR primers: sws2_300_Fw (5'- TCTTG TACTG CGCAG ATGTA G -3'), 250sw2_Fw (5'- GAAAC TTTGT GTGTA GCTGA TG -3'), and pGL4.13_Rv (5'- AGATC TGATA TCCTC GAGGC TAG -3'). For generating the pGL4 vector having no basal promoter, the SV40 promoter in the pGL4.13 vector was removed by a combination of enzyme digestion and self-ligation; the pGL4.13 vector was double digested with HindIII and Xhol, treated T4 DNA polymerase to make a blunt end, and self-ligated. The nucleotide mutations on the potential Foxq2-binding motif were introduced into the 0.25-kb SWS2 reporter by PCR. The promoter sequence in each of these resultant constructs was sequenced to confirm that no unintended mutation was incorporated into the promoter region.

To generate the expression plasmid of Foxq2, we first inserted a FLAG epitope tag into the pCAG vector (gifted from Dr. Takahiko Matsuda). The resultant vector was named as pCAG-FLAG. We then amplified the cDNA fragments of foxq2 from retinal cDNAs of adult zebrafish, and cloned them into the EcoRV-treated pCAG-FLAG vector. These plasmids was named as drFoxq2/pCAG-FLAG. For generating the VP64-Foxq2 expression plasmid, we first cloned the multiple repeats of the herpes simplex VP16 activation domain (synthesized DNA fragments) into the Xhol-treated pCAG-FLAG vector. The resultant plasmid, named as pCAG-FLAG-VP64N, was treated with the EcoRV and ligated with the PCR-amplified cDNA fragment. The nucleotide deletion in 
1 the DNA binding domain of Foxq2 was introduced by PCR. The DNA sequence

2 data for drFoxq2/pCAG-FLAG-VP64N will be available in the

3 DDBJ/EMBL/NCBI.

HEK293T17 cells were grown in the Dulbecco's modified Eagle's medium supplemented with $10 \%$ fetal bovine serum, 100 units $/ \mathrm{mL}$ penicillin, and 100 $\mu \mathrm{g} / \mathrm{mL}$ streptomycin. HEK293T17 cells in 24-well plates were transiently transfected with polyethyleneimine (Polysciences, \#24765). The firefly luciferase plasmid harboring the sws 2 promoter described above was used as a reporter plasmid (10 ng per well), while the Renilla luciferase plasmid, pGL4.74[hRluc/TK] (E6921, Promega), was simultaneously transfected as an internal control reporter ( $0.5 \mathrm{ng}$ per well). The amount of the expression plasmid of Foxq2 used for the transfection is indicated in the figure legends. The total amount of DNA transfected in each well was equally adjusted by adding the empty expression plasmids. The transfected cells were collected $36-48 \mathrm{~h}$ after the transfection, rinsed with PBS(-) (137 mM NaCl, $2.69 \mathrm{mM} \mathrm{KCl,} 5.5 \mathrm{mM} \mathrm{Na}-$ ${ }_{2} \mathrm{HPO}_{4}, 1.47 \mathrm{mM} \mathrm{KH}_{2} \mathrm{PO}_{4}$ ), and lysed with $100 \mu$ of Passive Lysis Buffer (Promega). Out of this lysate, $10 \mu \mathrm{l}$ was used for the dual luciferase assay, and the rest was further lysed in an SDS-PAGE sampling buffer for the immunoblot analysis described below. The dual luciferase assay was conducted with DualLuciferase $®$ Reporter Assay System (Promega) and a GloMax luminometer (Promega) according to the manufacturer's protocols. The luciferase activity derived from the firefly luciferase reporter was normalized to that from the

23 Renilla luciferase reporter.

Immunoblot analysis. Immunoblot analysis was carried out as described previously (23). In short, proteins lysed in an SDS-PAGE sampling buffer were separated on a gel by SDS-PAGE, transferred to a Immobilon-P transfer membrane (Millipore), and probed with primary antibodies overnight at $4^{\circ} \mathrm{C}$. The bound primary antibodies were detected by horseradish peroxidase-conjugated secondary antibodies in combination with an enhanced chemiluminescence detection system using Western Lightning Chemiluminescence Reagent (PerkinEImer Life Sciences) or ImmunoStar (Wako Pure Chemical Industries).

33 Chemiluminescent images were acquired with ImageQuant LAS 4000 mini (GE 
1 Healthcare). The primary antibodies used were as follows: anti-FLAG antibody

2 (F3165, Sigma, diluted to $0.8 \mu \mathrm{g} / \mathrm{ml}$ ); anti-H3K4me3 antibody (07-473, Upstate,

3 diluted 1:5000). The secondary antibodies used were as follows: horseradish

4 peroxidase-conjugated anti-mouse IgG (074-1816, Kirkegaard \& Perry

5 Laboratories, diluted to $0.2 \mu \mathrm{g} / \mathrm{ml}$ ) and horseradish peroxidase-conjugated antirabbit lgG (074-1516, Kirkegaard \& Perry Laboratories, diluted to $0.2 \mu \mathrm{g} / \mathrm{ml}$ ).

8 Generation of transgenic zebrafish. To construct a Foxq2 transgene 9 plasmid, the FLAG-Foxq2 coding sequence was amplified by PCR from the 10 plasmid, drFoxq2/pCAG-FLAG (described in the section of Luciferase assay).

11 The amplified fragment was then cloned with In-Fusion HD cloning kit into the pT2drCrx5.2kGP2ASix7 (23) digested by Xhol and BamHI. The resultant

13 plasmid, named pT2drCrx5.2kGP2AFoxq2, was used for the generation of 14 transgenic zebrafish. The foxq2 transgenic zebrafish, ja78Tg, ja79Tg, and 15 ja91Tg strains, were generated with the Tol2-based transgenesis system (61).

16 The Tol2 transposase mRNA was transcribed from pCS-TP in vitro using the 17 SP6 mMESSAGE mMACHINE Kit (Ambion) and purified with the RNeasy Mini kit (Qiagen). The purified Tol2 mRNA and the plasmid DNA were mixed and diluted to a final concentration of $25 \mathrm{ng} / \mu \mathrm{l}$ for each in $0.05 \%$ phenol red solution. About $1 \mathrm{nl}$ of the DNA/RNA solution was injected into each of wild-type embryos at the one-cell stage. Fluorescence-positive embryos were isolated and raised to adulthood. The raised F0 founder fish were crossed with the wild-type fish, and subsequent F1 embryos were screened by the presence of fluorescence at four or five days post fertilization (dpf). The transgenic lines were established from individual F0 fish. The foxq2-tg larval fish and its siblings were genotyped by ocular EGFP fluorescence just before the sampling, and soaked in RNAlater for RT-qPCR analysis or fixed with 4\% PFA in D-PBS for cryosectioning. The GFP-negative siblings were used as a control. To unambiguously identify the transgenic larvae according to ocular EGFP expression, synthesis of melanin pigment was inhibited by treating embryos with the egg water containing 
1 Motif scanning. The sws2 promoter (Fig. S4) was sequence-verified by

2 traditional Sanger sequencing and used for the motif scanning. Fox and Crx

3 binding profiles, each represented as a matrix consisting of nucleotide counts

4 per position, i.e. position frequency matrix (PFM), were retrieved from the

5 JASPAR CORE database (http://jaspar.genereg.net) (62). These retrieved

6 binding profiles (total eleven profiles) were composed of one binding profile of

7 Crx in mouse (63) and two binding profiles (FkhP and FkhS) for each of five Fox

8 proteins in mouse (FoxA2, FoxL1, FoxK1, FoxJ1, and FoxJ3) (64). These

9 frequency matrices were used to construct position-dependent letter-probability

10 matrices that describe the probability of each possible letter at each position in

11 the pattern with the simplest background model assuming that each letter

12 appears equally frequently in the dataset. The sws 2 promoter was scanned for

13 individual matches to each of the Crx and Fox motifs with FIMO v5.1.1 (65).

14 Biased distribution of individual letters in the promoter sequences was

15 normalized by a 0 -order model of Markov background probabilities constructed

16 with the fasta-get-Markov tool in the MEME suite v5.1.1 (65) using nucleotide

17 sequences between $1000 \mathrm{bp}$ upstream and $1000 \mathrm{bp}$ downstream of a

18 transcription start site for all protein-coding genes in zebrafish (genome

19 assembly GRCz11, Ensembl Release 98). All motif occurrences with a P-value

20 less than $1 \times 10^{-4}$ are indicated in Fig. S4. The motif scanning results and matrix

21 IDs of Fox and Crx in the database are included in Data S2.

23 BLAST searches and phylogenetic analysis. For identifying FOXQ2 genes

in platypus and chicken, tBLASTn search (Ensembl web tools) was conducted against genome sequences of platypus (Reference Genome ID: mOrnAna1.p.v1) and chicken (Reference Genome ID: GRCg6a) using the amino acid sequence of forkhead domain of zebrafish Foxq2 (Ensembl protein ID: ENSDARP00000119225.2) as the query. We retrieved nucleotide sequences in intergenic regions showing the higher alignment score than any other regions encoding members of Fox families. The retrieved nucleotide sequences of FOXQ2 genes were mapped onto two distinct genomic regions, but were adjacently located in the region of the same chromosome, where any other gene is not annotated. We thus assumed that these mapped regions are 
1 two exons of FOXQ2 genes. Consistently, the forkhead domain of zebrafish

2 Foxq2 is encoded in two exons. We then manually annotated exon-exon

3 junctions of FOXQ2 genes according to the GT/AG mRNA processing rule. The

4 annotated cDNA sequence of the forkhead domain of FOXQ2 gene was

5 translated into a protein sequence and used for constructing a phylogenetic tree

6 described in the next paragraph. The nucleotide sequences and annotations of

7 FOXQ2 genes are summarized in Data S3.

For constructing a phylogenetic tree, FOXQ2 amino acid sequences in

9 spotted gar, medaka, coelacanth, and sparrow hawk were retrieved from

10 Ensembl data base (Ensembl Release 101). FOXQ2 sequences in platypus and

11 chicken identified by our blast searching were also used for the phylogenetic

12 tree construction. Amino acid sequences of other members of Fox subfamilies

13 were retrieved by performing tblastn searches (NCBI) against RefSeq RNA

14 transcripts in purple sea urchin, zebrafish, chicken, platypus, and human using

15 the amino acid sequence of forkhead domain of zebrafish Foxq2 as the query

16 sequence with E-values $<1 e-20$. Among the retrieved cDNA sequences,

17 members of representative Fox subfamily (FOXA, FOXB, FOXC, FOXF, FOXJ

18 and FOXQ) were selected and translated into amino acid sequences. The

19 resultant sequences of Fox proteins were aligned by multiple sequence

20 alignment programs: G-INS-i program in MAFFT v7.471 under default settings

21 (66). The aligned sequences were trimmed, remaining the sequences of the

22 forkhead domain. Alignment gaps were manually inspected and deleted. The

23 maximum likelihood tree was inferred by RAxML-NG v1.0.2 (67). The best tree

24 was selected out of forty alternative runs on twenty random and twenty

25 parsimony-based starting trees (--tree pars $\{20\}$, rand $\{20\}$ option). The amino

26 acid replacement models of Le-Gascuel (LG) with gamma distribution (G4) were

27 selected using the Akaike information criterion implemented in ModelTest-NG

28 version x.y.z (68). The bootstrap values were obtained from sampling 500

29 times. The amino acid sequences used for the construction of phylogenetic tree

30 are listed in Data S3. Accession numbers for genome assemblies and Fox

31 genes are also provided in Data S3. 
1 Statistical analysis. Sample sizes were determined based on prior literature 2 and best practices in the field, and no statistical methods were used to 3 predetermine sample size. A two-tailed unpaired $t$-test was used to determine 4 the statistical significance between two datasets (Excel). Tukey-Kramer 5 honestly significant difference test was used to determine the statistical 6 significance among multiple datasets (the 'stats' package in R, version 3.6.1). 7 


\section{References}

1. T. D. Lamb, Evolution of phototransduction, vertebrate photoreceptors and retina. Progress in Retinal and Eye Research. 36, 52-119 (2013).

2. T. H. Goldsmith, Optimization, constraint, and history in the evolution of eyes. The Quarterly review of biology. 65, 281-322 (1990).

3. T. Baden, D. Osorio, The Retinal Basis of Vertebrate Color Vision. Annual Review of Vision Science. 5, 177-200 (2019).

4. T. Okano, D. Kojima, Y. Fukada, Y. Shichida, T. Yoshizawa, Primary structures of chicken cone visual pigments: vertebrate rhodopsins have evolved out of cone visual pigments. PNAS. 89, 5932-5936 (1992).

5. S. Yokoyama, Evolution of Dim-Light and Color Vision Pigments. Annual Review of Genomics and Human Genetics. 9, 259-282 (2008).

6. S. P. Collin, M. A. Knight, W. L. Davies, I. C. Potter, D. M. Hunt, A. E. O. Trezise, Ancient colour vision: multiple opsin genes in the ancestral vertebrates. Current Biology. 13, R864-R865 (2003).

7. W. I. L. Davies, S. P. Collin, D. M. Hunt, Molecular ecology and adaptation of visual photopigments in craniates. Molecular Ecology. 21, 3121-3158 (2012).

8. A. Swaroop, D. Kim, D. Forrest, Transcriptional regulation of photoreceptor development and homeostasis in the mammalian retina. Nat Rev Neurosci. 11, 563-576 (2010).

9. K. Viets, K. C. Eldred, R. J. Johnston, Mechanisms of Photoreceptor Patterning in Vertebrates and Invertebrates. Trends in Genetics. 32, 638659 (2016).

10. C. Cepko, Intrinsically different retinal progenitor cells produce specific types of progeny. Nat Rev Neurosci. 15, 615-627 (2014).

11. T. Furukawa, E. M. Morrow, C. L. Cepko, Crx, a Novel otx-like Homeobox Gene, Shows Photoreceptor-Specific Expression and Regulates Photoreceptor Differentiation. Cell. 91, 531-541 (1997).

12. C. L. Freund, C. Y. Gregory-Evans, T. Furukawa, M. Papaioannou, J. Looser, L. Ploder, J. Bellingham, D. Ng, J.-A. S. Herbrick, A. Duncan, S. W. Scherer, L.-C. Tsui, A. Loutradis-Anagnostou, S. G. Jacobson, C. L. Cepko, S. S. Bhattacharya, R. R. McInnes, Cone-Rod Dystrophy Due to Mutations in a Novel Photoreceptor-Specific Homeobox Gene (CRX) Essential for Maintenance of the Photoreceptor. Cell. 91, 543-553 (1997).

13. A. J. Mears, M. Kondo, P. K. Swain, Y. Takada, R. A. Bush, T. L. Saunders, P. A. Sieving, A. Swaroop, Nrl is required for rod photoreceptor development. Nature Genetics. 29, 447-452 (2001). 
14. N. B. Haider, J. Naggert, P. M. Nishina, Excess cone cell proliferation due to lack of a functional NR2E3 causes retinal dysplasia and degeneration in rd7/rd7 mice. Hum Mol Genet. 10, 1619-1626 (2001).

15. L. Ng, J. B. Hurley, B. Dierks, M. Srinivas, C. Saltó, B. Vennström, T. A. Reh, D. Forrest, A thyroid hormone receptor that is required for the development of green cone photoreceptors. Nat Genet. 27, 94-98 (2001).

16. S. C. Suzuki, A. Bleckert, P. R. Williams, M. Takechi, S. Kawamura, R. O. L. Wong, Cone photoreceptor types in zebrafish are generated by symmetric terminal divisions of dedicated precursors. PNAS. 110, 1510915114 (2013).

17. K. C. Eldred, S. E. Hadyniak, K. A. Hussey, B. Brenerman, P.-W. Zhang, X. Chamling, V. M. Sluch, D. S. Welsbie, S. Hattar, J. Taylor, K. Wahlin, D. J. Zack, R. J. Johnston, Thyroid hormone signaling specifies cone subtypes in human retinal organoids. Science. 362 (2018), doi:10.1126/science.aau6348.

18. K. Alvarez-Delfin, A. C. Morris, C. D. Snelson, J. T. Gamse, T. Gupta, F. L. Marlow, M. C. Mullins, H. A. Burgess, M. Granato, J. M. Fadool, Tbx2b is required for ultraviolet photoreceptor cell specification during zebrafish retinal development. PNAS. 106, 2023-2028 (2009).

19. Y. Ogawa, T. Shiraki, D. Kojima, Y. Fukada, Homeobox transcription factor Six7 governs expression of green opsin genes in zebrafish. Proceedings of the Royal Society B: Biological Sciences. 282, 20150659 (2015).

20. M. Sotolongo-Lopez, K. Alvarez-Delfin, C. J. Saade, D. L. Vera, J. M. Fadool, Genetic Dissection of Dual Roles for the Transcription Factor six7 in Photoreceptor Development and Patterning in Zebrafish. PLOS Genetics. 12, e1005968 (2016).

21. A. Chinen, T. Hamaoka, Y. Yamada, S. Kawamura, Gene Duplication and Spectral Diversification of Cone Visual Pigments of Zebrafish. Genetics. 163, 663-675 (2003).

22. M. Takechi, S. Kawamura, Temporal and spatial changes in the expression pattern of multiple red and green subtype opsin genes during zebrafish development. Journal of Experimental Biology. 208, 1337-1345 (2005).

23. Y. Ogawa, T. Shiraki, Y. Asano, A. Muto, K. Kawakami, Y. Suzuki, D. Kojima, Y. Fukada, Six6 and Six7 coordinately regulate expression of middle-wavelength opsins in zebrafish. PNAS. 116, 4651-4660 (2019).

24. C. W. Müller, B. G. Herrmann, Crystallographic structure of the T domainDNA complex of the Brachyury transcription factor. Nature. 389, 884-888 (1997). 
25. C. D. Snelson, K. Santhakumar, M. E. Halpern, J. T. Gamse, Tbx2b is required for the development of the parapineal organ. Development. 135, 1693-1702 (2008).

26. L. I. Volkov, J. S. Kim-Han, L. M. Saunders, D. Poria, A. E. O. Hughes, V. J. Kefalov, D. M. Parichy, J. C. Corbo, Thyroid hormone receptors mediate two distinct mechanisms of long-wavelength vision. PNAS. 117, 1526215269 (2020).

27. C. Deveau, X. Jiao, S. C. Suzuki, A. Krishnakumar, T. Yoshimatsu, J. F. Hejtmancik, R. F. Nelson, Thyroid hormone receptor beta mutations alter photoreceptor development and function in Danio rerio (zebrafish). PLOS Genetics. 16, e1008869 (2020).

28. H. Nunley, M. Nagashima, K. Martin, A. L. Gonzalez, S. C. Suzuki, D. A. Norton, R. O. L. Wong, P. A. Raymond, D. K. Lubensky, Defect patterns on the curved surface of fish retinae suggest a mechanism of cone mosaic formation. PLOS Computational Biology. 16, e1008437 (2020).

29. J. M. Gross, J. E. Dowling, Tbx2b is essential for neuronal differentiation along the dorsal/ventral axis of the zebrafish retina. PNAS. 102, 43714376 (2005).

30. K. L. Clark, E. D. Halay, E. Lai, S. K. Burley, Co-crystal structure of the HNF-3/ fork head DNA-recognition motif resembles histone H5. Nature. 364, 412-420 (1993).

31. C. Larroux, G. N. Luke, P. Koopman, D. S. Rokhsar, S. M. Shimeld, B. M. Degnan, Genesis and Expansion of Metazoan Transcription Factor Gene Classes. Mol Biol Evol. 25, 980-996 (2008).

32. S. Nakagawa, S. S. Gisselbrecht, J. M. Rogers, D. L. Hartl, M. L. Bulyk, DNA-binding specificity changes in the evolution of forkhead transcription factors. PNAS. 110, 12349-12354 (2013).

33. M. Takechi, S. Seno, S. Kawamura, Identification of cis-Acting Elements Repressing Blue Opsin Expression in Zebrafish UV Cones and Pineal Cells. J. Biol. Chem. 283, 31625-31632 (2008).

34. R. R. Beerli, D. J. Segal, B. Dreier, C. F. Barbas, Toward controlling gene expression at will: Specific regulation of the erbB-2/HER-2 promoter by using polydactyl zinc finger proteins constructed from modular building blocks. PNAS. 95, 14628-14633 (1998).

35. S. L. Renninger, M. Gesemann, S. C. F. Neuhauss, Cone arrestin confers cone vision of high temporal resolution in zebrafish larvae. European Journal of Neuroscience. 33, 658-667 (2011).

36. W. L. Davies, L. S. Carvalho, J. A. Cowing, L. D. Beazley, D. M. Hunt, C. A. Arrese, Visual pigments of the platypus: A novel route to mammalian colour vision. Current Biology. 17, R161-R163 (2007). 
37. J. K. Bowmaker, Evolution of vertebrate visual pigments. Vision Research. 48, 2022-2041 (2008).

38. G. H. Jacobs, Evolution of colour vision in mammals. Philosophical Transactions of the Royal Society B: Biological Sciences. 364, 2957-2967 (2009).

39. T. W. Cronin, M. J. Bok, Photoreception and vision in the ultraviolet. Journal of Experimental Biology. 219, 2790-2801 (2016).

40. T. Yoshimatsu, P. Bartel, C. Schröder, F. K. Janiak, F. St-Pierre, P. Berens, T. Baden, bioRxiv, in press, doi:10.1101/2020.10.26.356089.

41. O. Hobert, Regulatory logic of neuronal diversity: Terminal selector genes and selector motifs. PNAS. 105, 20067-20071 (2008).

42. Y. Liu, Y.-C. Shen, J. S. Rest, P. A. Raymond, D. J. Zack, Isolation and Characterization of a Zebrafish Homologue of the Cone Rod Homeobox Gene. Invest. Ophthalmol. Vis. Sci. 42, 481-487 (2001).

43. T. Ebrey, Y. Koutalos, Vertebrate Photoreceptors. Progress in Retinal and Eye Research. 20, 49-94 (2001).

44. J. H. Fritzenwanker, J. Gerhart, R. M. Freeman, C. J. Lowe, The Fox/Forkhead transcription factor family of the hemichordate Saccoglossus kowalevskii. EvoDevo. 5, 17 (2014).

45. C. Sinigaglia, H. Busengdal, L. Leclère, U. Technau, F. Rentzsch, The Bilaterian Head Patterning Gene six3/6 Controls Aboral Domain Development in a Cnidarian. PLOS Biology. 11, e1001488 (2013).

46. R. C. Range, Z. Wei, An anterior signaling center patterns and sizes the anterior neuroectoderm of the sea urchin embryo. Development. 143, 1523-1533 (2016).

47. T. D. Lamb, S. P. Collin, E. N. Pugh, Evolution of the vertebrate eye: opsins, photoreceptors, retina and eye cup. Nature Reviews Neuroscience. 8, 960-976 (2007).

48. D. Arendt, K. Tessmar-Raible, H. Snyman, A. W. Dorresteijn, J. Wittbrodt, Ciliary Photoreceptors with a Vertebrate-Type Opsin in an Invertebrate Brain. Science. 306, 869-871 (2004).

49. Y. Asaoka, H. Mano, D. Kojima, Y. Fukada, Pineal expression-promoting element (PIPE), a cis-acting element, directs pineal-specific gene expression in zebrafish. PNAS. 99, 15456-15461 (2002).

50. H. Mano, Y. Asaoka, D. Kojima, Y. Fukada, Brain-specific homeobox Bsx specifies identity of pineal gland between serially homologous photoreceptive organs in zebrafish. Communications Biology. 2, 1-10 (2019). 
51. M. Takechi, T. Hamaoka, S. Kawamura, Fluorescence visualization of ultraviolet-sensitive cone photoreceptor development in living zebrafish. FEBS Letters. 553, 90-94 (2003).

52. T. Tsujimura, A. Chinen, S. Kawamura, Identification of a locus control region for quadruplicated green-sensitive opsin genes in zebrafish. PNAS. 104, 12813-12818 (2007).

53. T. Tsujimura, T. Hosoya, S. Kawamura, A Single Enhancer Regulating the Differential Expression of Duplicated Red-Sensitive Opsin Genes in Zebrafish. PLOS Genetics. 6, e1001245 (2010).

54. Y. Naito, K. Hino, H. Bono, K. Ui-Tei, CRISPRdirect: software for designing CRISPR/Cas guide RNA with reduced off-target sites. Bioinformatics. 31, 1120-1123 (2015).

55. T. G. Montague, J. M. Cruz, J. A. Gagnon, G. M. Church, E. Valen, CHOPCHOP: a CRISPR/Cas9 and TALEN web tool for genome editing. Nucleic Acids Res. 42, W401-W407 (2014).

56. M. A. Moreno-Mateos, C. E. Vejnar, J.-D. Beaudoin, J. P. Fernandez, E. K. Mis, M. K. Khokha, A. J. Giraldez, CRISPRscan: designing highly efficient sgRNAs for CRISPR-Cas9 targeting in vivo. Nat Methods. 12, 982-988 (2015).

57. J. A. Gagnon, E. Valen, S. B. Thyme, P. Huang, L. Ahkmetova, A. Pauli, T. G. Montague, S. Zimmerman, C. Richter, A. F. Schier, Efficient Mutagenesis by Cas9 Protein-Mediated Oligonucleotide Insertion and Large-Scale Assessment of Single-Guide RNAs. PLOS ONE. 9, e98186 (2014).

58. L.-E. Jao, S. R. Wente, W. Chen, Efficient multiplex biallelic zebrafish genome editing using a CRISPR nuclease system. PNAS. 110, 1390413909 (2013).

59. S. Ansai, M. Kinoshita, Targeted mutagenesis using CRISPR/Cas system in medaka. Biology Open. 3, 362-371 (2014).

60. S. Ota, Y. Hisano, M. Muraki, K. Hoshijima, T. J. Dahlem, D. J. Grunwald, Y. Okada, A. Kawahara, Efficient identification of TALEN-mediated genome modifications using heteroduplex mobility assays. Genes to Cells. 18, 450-458 (2013).

61. K. Kawakami, H. Takeda, N. Kawakami, M. Kobayashi, N. Matsuda, M. Mishina, A Transposon-Mediated Gene Trap Approach Identifies Developmentally Regulated Genes in Zebrafish. Developmental Cell. 7, 133-144 (2004).

62. O. Fornes, J. A. Castro-Mondragon, A. Khan, R. van der Lee, X. Zhang, P. A. Richmond, B. P. Modi, S. Correard, M. Gheorghe, D. Baranašić, W. Santana-Garcia, G. Tan, J. Chèneby, B. Ballester, F. Parcy, A. Sandelin, 
B. Lenhard, W. W. Wasserman, A. Mathelier, JASPAR 2020: update of the open-access database of transcription factor binding profiles. Nucleic Acids Research. 48, D87-D92 (2020).

63. J. C. Corbo, K. A. Lawrence, M. Karlstetter, C. A. Myers, M. Abdelaziz, W. Dirkes, K. Weigelt, M. Seifert, V. Benes, L. G. Fritsche, B. H. F. Weber, T. Langmann, CRX ChIP-seq reveals the cis-regulatory architecture of mouse photoreceptors. Genome Res. 20, 1512-1525 (2010).

64. G. Badis, M. F. Berger, A. A. Philippakis, S. Talukder, A. R. Gehrke, S. A. Jaeger, E. T. Chan, G. Metzler, A. Vedenko, X. Chen, H. Kuznetsov, C.-F. Wang, D. Coburn, D. E. Newburger, Q. Morris, T. R. Hughes, M. L. Bulyk, Diversity and Complexity in DNA Recognition by Transcription Factors. Science. 324, 1720-1723 (2009).

65. T. L. Bailey, M. Boden, F. A. Buske, M. Frith, C. E. Grant, L. Clementi, J. Ren, W. W. Li, W. S. Noble, MEME Suite: tools for motif discovery and searching. Nucleic Acids Research. 37, W202-W208 (2009).

66. K. Katoh, D. M. Standley, MAFFT Multiple Sequence Alignment Software Version 7: Improvements in Performance and Usability. Molecular Biology and Evolution. 30, 772-780 (2013).

67. A. M. Kozlov, D. Darriba, T. Flouri, B. Morel, A. Stamatakis, RAxML-NG: a fast, scalable and user-friendly tool for maximum likelihood phylogenetic inference. Bioinformatics. 35, 4453-4455 (2019).

68. D. Darriba, D. Posada, A. M. Kozlov, A. Stamatakis, B. Morel, T. Flouri, ModelTest-NG: A New and Scalable Tool for the Selection of DNA and Protein Evolutionary Models. Molecular Biology and Evolution. 37, 291294 (2020).

69. J. Li, A. C. Dantas Machado, M. Guo, J. M. Sagendorf, Z. Zhou, L. Jiang, X. Chen, D. Wu, L. Qu, Z. Chen, L. Chen, R. Rohs, Y. Chen, Structure of the Forkhead Domain of FOXA2 Bound to a Complete DNA Consensus Site. Biochemistry. 56, 3745-3753 (2017).

70. D. Lagman, D. Ocampo Daza, J. Widmark, X. M. Abalo, G. Sundström, D. Larhammar, The vertebrate ancestral repertoire of visual opsins, transducin alpha subunits and oxytocin/vasopressin receptors was established by duplication of their shared genomic region in the two rounds of early vertebrate genome duplications. BMC Evolutionary Biology. 13, 238 (2013). 


\section{Acknowledgements}

We are grateful to members of the Fukada lab for valuable discussion, especially to M. Nagata for technical assistance. We thank the National

4 BioResource Project (NBRP) Zebrafish for providing the transgenic line, and Dr.

5 Shoji Kawamura, the University of Tokyo, for the zebrafish opsin transgenic

6 lines, $T g(-5.5 o p n 1 s w 1: E G F P)^{\mathrm{kj} 9}, T g(-3.5 o p n 1 s w 2: E G F P)^{\mathrm{kj} 11}, \operatorname{Tg}(R H 2-2 / G F P$ -

$7 \quad P A C)^{\mathrm{kj} 4}$ and $T g(-0.6 o p n 1 / w 1-/ w s 2: G F P)^{\mathrm{kj} 19}$. We also thank Dr. Takahiko

8 Matsuda for providing the pCAG empty vector, Dr. Masato Kinoshita for the

9 pCS2+hSpCas9 vector, and Dr. Wenbiao Chen for the pCS2-nCas9n vector.

10 We also thank members of the FACS core laboratory, the University of Tokyo,

11 for helping sorting cells. This work was supported in part by JSPS KAKENHI

12 Grant Numbers JP16J01681 (to Y.O.), JP19K16196 (to T.S.), JP19K06758 (to

13 D.K.) and JP17H06096 (to Y.F.) and also by a research grant from Research

14 Foundation for Opto-Science and Technology (to D.K.). Y.O., T.S., D.K., and

15 Y.F. conceived and designed the research. Y.O. and T.S. constructed plasmids

16 and generated zebrafish lines. Y.O conducted all the other molecular lab work

17 and computational analysis. D.K. and Y.F. supervised the project. Y.O., D.K.,

18 and Y.F. wrote the manuscript, and T.S. contributed to preparation of the draft.

19 All authors approved the manuscript for publication. The authors declare that

20 they have no competing interests. All data needed to evaluate the conclusions

21 in the paper are present in the paper and/or the Supplementary Materials. 
A

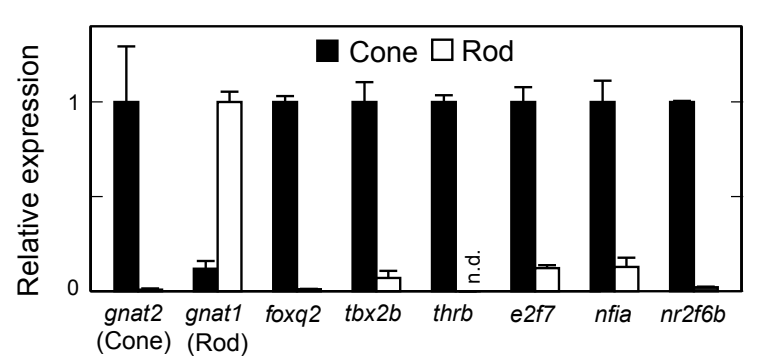

C

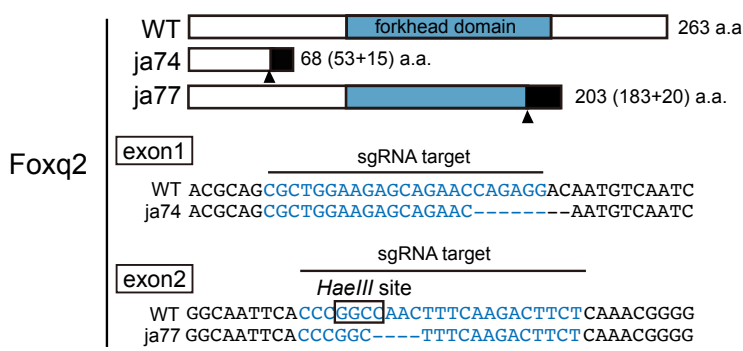

E

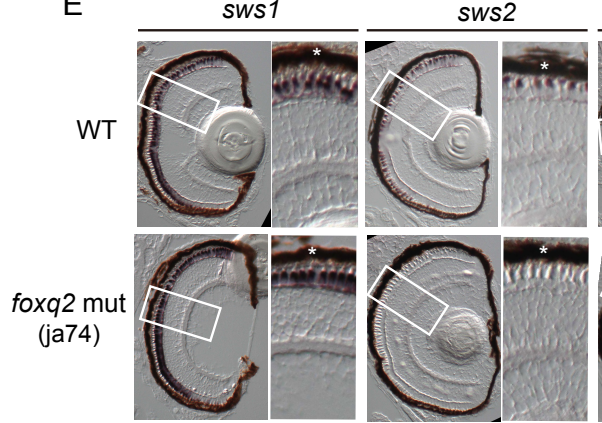

$\mathrm{F}$

Tbx2b

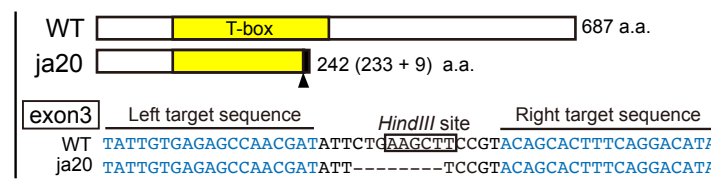

$\mathrm{H}$

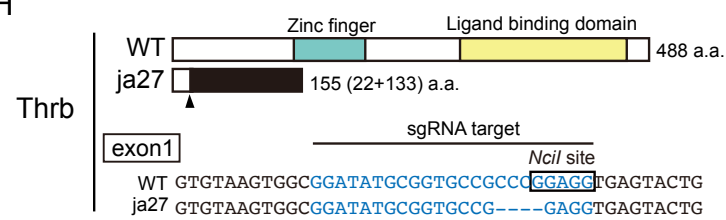

B
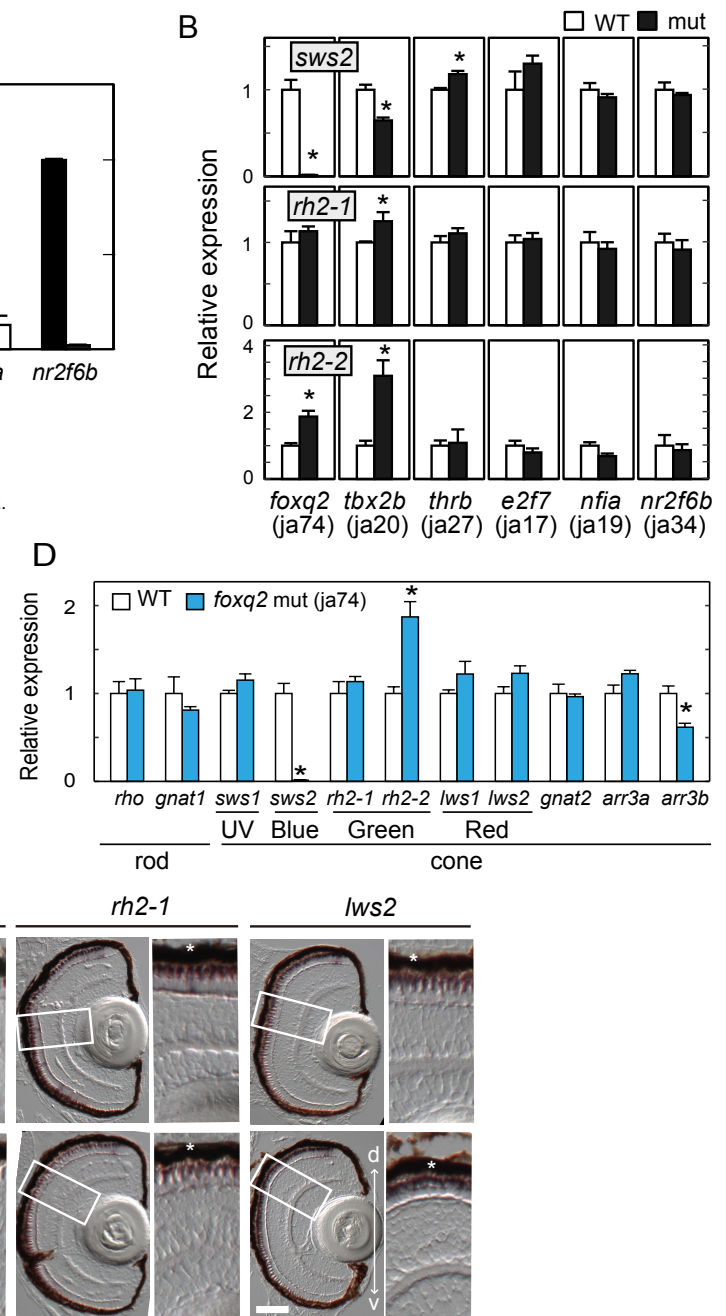

G

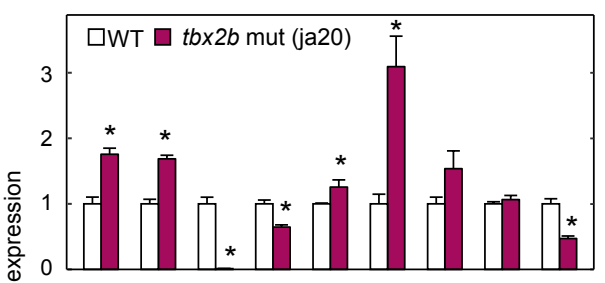

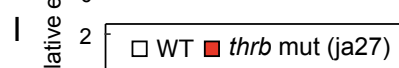

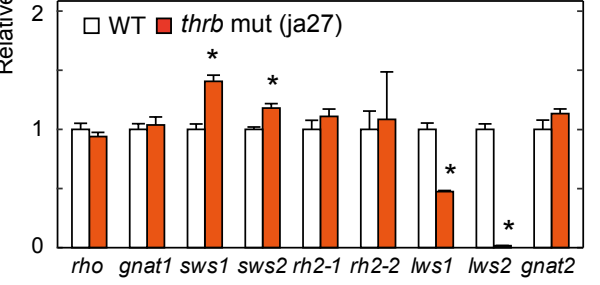

\section{Fig. 1 The loss of function analysis for cone-enriched transcription}

factors. (A) Relative expression levels of phototransduction genes and

transcription factors in isolated rods and cones at the adult stage (Mean $\pm S D, n$

7 = 2). n.d., not detected. (B) Relative expression levels of sws2 opsin in the 
1 larval eyes at five days post fertilization (dpf). Mean \pm SEM. ${ }^{*} P<0.05$ by

2 Student's $t$-test. The number of fish used was as follows: $n=5$ (foxq2 WT), $n=$

35 (foxq2 mut); $n=5$ (tbx2b WT), $n=5$ (tbx2b mut); $n=3$ (thrb WT), $n=4$ (thrb

4 mut); $n=4$ (e2f7 WT), $n=4$ (e2f7 mut); $n=3$ (nfia WT), $n=4$ (nfia mut); $n=4$

5 (nr2f6b WT), $n=4$ (nr2f6b mut). See also Fig. S3. (C, F, H) Schematic

6 representation of Foxq2, Tbx2b and Thrb, and their partial nucleotide

7 sequences. The frameshift site is indicated by an arrowhead. Nucleotide

8 deletions are indicated by dashes. The nucleotide sequences (letters in blue)

9 indicate the target sequences of TAL-effector nucleases or Cas9-sgRNA

10 complexes. The recognition sites of the restriction endonucleases, Ncil, HindlII

11 and Haelll, are surrounded by black lines. The ja74 and ja77 mutation caused a

12 frame shift of the amino acid sequence of Foxq2 by 8-bp and 4-bp loss,

13 respectively. The ja20 mutation caused a frame shift of the amino acid

14 sequence of Tbx2b by 8-bp loss. The ja27 mutation (4-bp loss) caused a frame

15 shift of the amino acid sequence of Thrb2, which is an isoform of Thrb and

16 essential for LWS opsin expression in mice and zebrafish $(15,16)$. (D, G, I)

17 Expression profiles of phototransduction genes in the 5 dpf-larval eyes. Mean \pm

18 SEM. * $P<0.05$ by Student's $t$-test. The number of fish used was as follows: $n=$

195 (foxq2 WT), $n=5$ (foxq2 mut); $n=5$ (tbx2b WT), $n=5$ (tbx2b mut); $n=3$ (thrb

$20 \mathrm{WT}$ ), $n=4$ (thrb mut). The expression levels of sws2 and $r h 2$ genes are

21 reproduced in the panel $B$. (E) Expression pattern of cone opsin genes

22 examined by in situ hybridization using 5-dpf larval eyes of the foxq2 mut (ja74).

23 Magnified view (a box surrounded with white lines) is indicated in the right side

24 of each panel. The retinal pigmented epithelium (RPE, indicated by asterisks) is 25 adjacent to the photoreceptor layer. $d-v$, dorsal-ventral retina. Scale bar, $50 \mu \mathrm{m}$. 

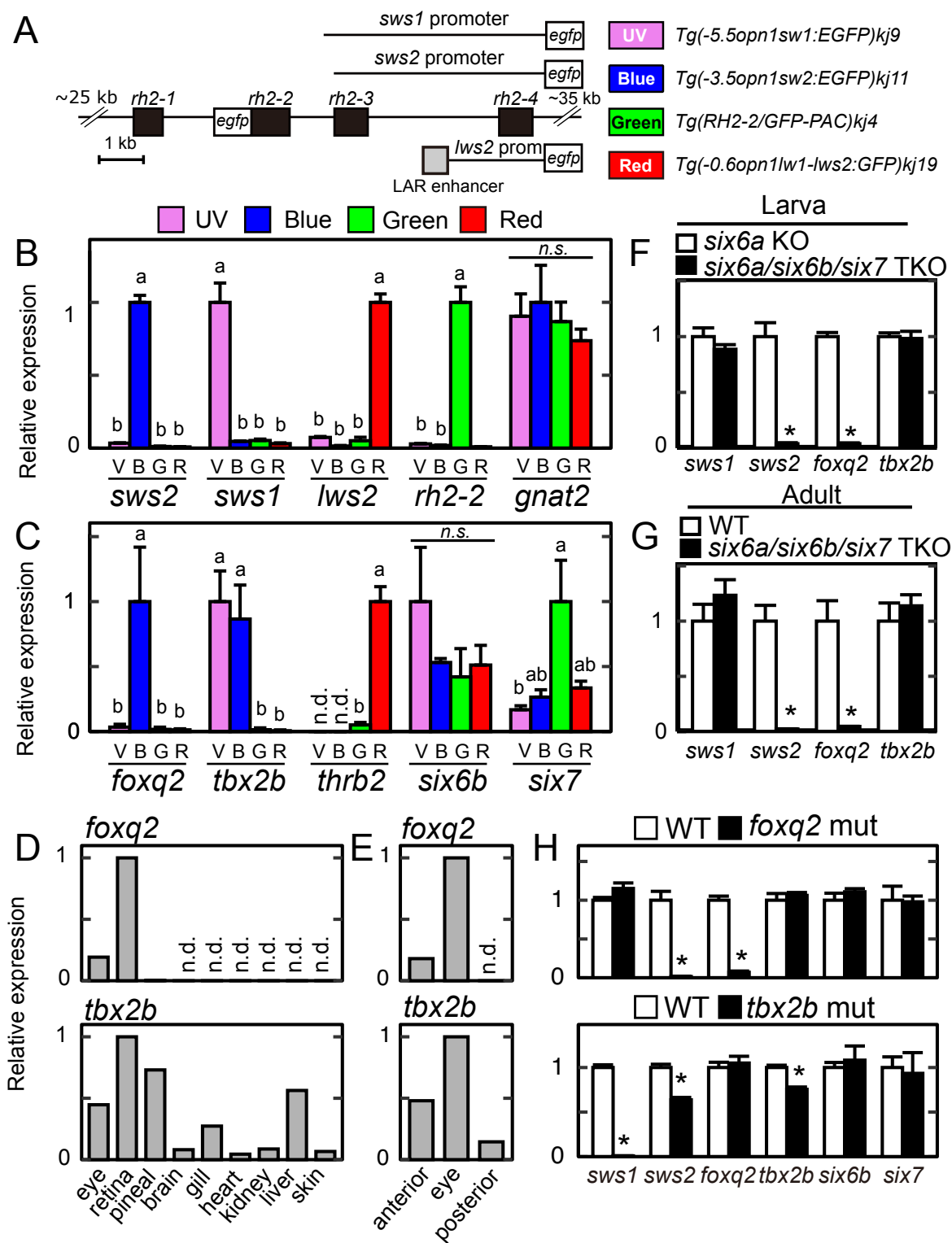

3 Fig. 2 Gene expression profiles of $t b \times 2 b$ and foxq2. (A) Schematic drawing

4 of transgenes for the four transgenic lines each expressing EGFP in SWS1

5 (UV), SWS2 (Blue), RH2 (Green) or LWS (Red) cone subtype. See also

6 materials and methods. (B, C) Relative expression levels of cone opsin genes

7 (B) and transcription factors (C) among isolated cone subtypes at the adult

8 stage. Mean expression values with SEM $(n=3)$ are indicated as bars. Distinct

9 letters indicate statistically significant differences $(P<0.05$ by Tukey's multiple

10 comparison test). n.d., not detected. n.s., not significant. (D, E) Relative

11 expression levels of $t b \times 2 b$ and foxq2 in adult tissues (D), 4-dpf larval anterior

12 segments, posterior segments and eyes (E). n.d., not detected. (F) Relative 
1 expression levels of $t b x 2 b$ and foxq 2 in the 5-dpf larval eyes of the

2 six6a/six6b/six7 TKO. Mean $\pm \operatorname{SEM}(n=5)$. ${ }^{*} P<0.05$ by Student's $t$-test. Note

3 that the six6a $\mathrm{KO}$ showed similar levels of cone opsin gene expression as

4 compared to the wild-type [See Ref. (23)]. (G) Relative expression levels of

$5 t b \times 2 b$ and foxq2 in the adult eyes of the six6a/six6b/six7 TKO. All of the fish

6 used here are in the transgenic background, Tg(-3.5opn1sw2:EGFP) ${ }^{k 111 T g}$,

7 where EGFP is expressed in the SWS2 cone subtype. Data are represented by

8 mean \pm SD ( $n=3$, WT; $n=2$, six6a/six6b/six7 TKO). $(\mathrm{H})$ Relative expression

9 levels of cone opsins and transcription factors responsible for photoreceptor

10 gene expression in the eyes of the $t b \times 2 b$ (ja20) and foxq2 (ja74) mutants. Mean

$11 \pm$ SEM. ${ }^{*} P<0.05$ by Student's $t$-test. The number of fish used was as follows: $n$

$12=5$ (tbx2b WT), $n=4$ (tbx2b mut); $n=5$ (foxq2 WT), $n=5$ (foxq2 mut). 
A

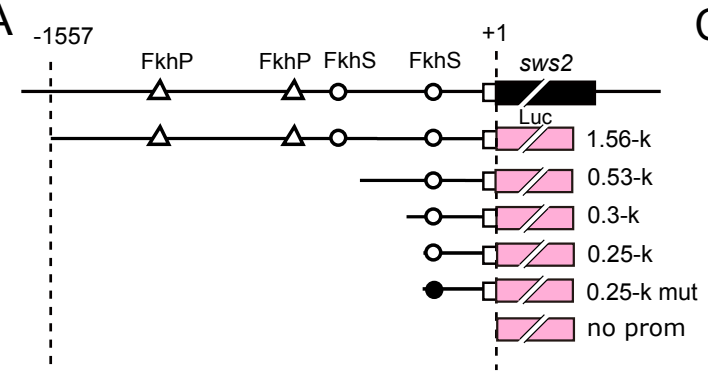

B

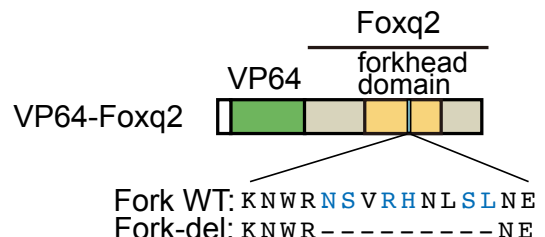

E

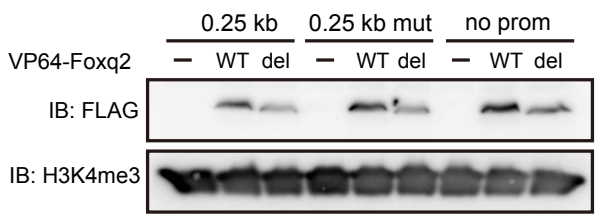

C

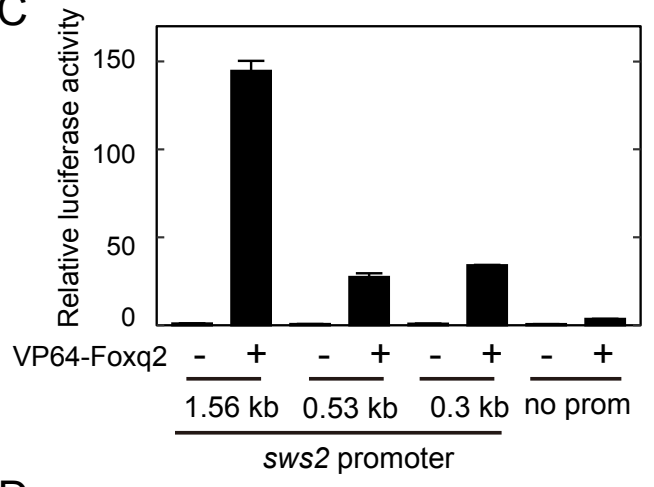

D

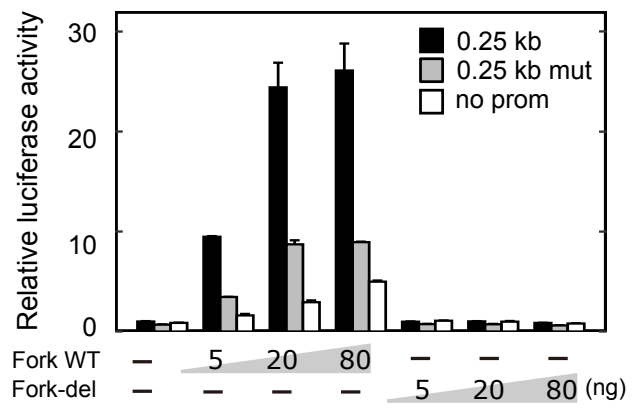

Fig. 3 DNA binding of Foxq2 to sws2 promoter. (A) Schematic structure of the promoter-luciferase reporter constructs for zebrafish sws2. The coding (solid box) and the untranslated (open box) regions of the first exon are indicated. The translational start site is marked as +1 . The forkhead binding motif (ACAACA) and its mutant (GTGGTG) are marked as open and closed circles, respectively. See also Fig. S4. (B) Schematic representation of VP64-Foxq2 protein. The Foxq2 mutant protein (Fork-del) lacks the canonical Fox base-contacting residues in the forkhead domain. These critical amino acid residues are highlighted in blue according to the previous paper (32). (C, D) The transcriptional assay in HEK293T17 cells using the luciferase reporter containing the promoter region of zebrafish sws2. The luciferase activity derived from the firefly luciferase reporter was normalized to that from the Renilla luciferase reporter. Data are represented by the mean $\pm \operatorname{SD}(n=2)$.

(C) The luciferase activity in each condition was normalized to the mean value obtained from the cells transfected with both empty vector and 1.56-kb sws2 reporter vector (leftmost bar). VP64-Foxq2 expression plasmid (100 ng) was used for

20 the transfection. (D) The amount of VP64-Foxq2 expression plasmid used for each well is indicated in the graph. The luciferase activity in each condition was 
1 normalized to the mean value obtained from the cells transfected with both the 2 empty expression vector and the $0.25-\mathrm{kb}$ sws2 $(0.25 \mathrm{~kb})$ reporter vector

3 (leftmost bar). (E) Protein expression of VP64-Foxq2 (WT) and its mutant

4 protein (Fork del) in the HEK293T17 cells transfected with $80 \mathrm{ng}$ of the

5 expressing plasmid. The antibody against a histone modification (H3K4me3) is

6 served as a loading control.

7 


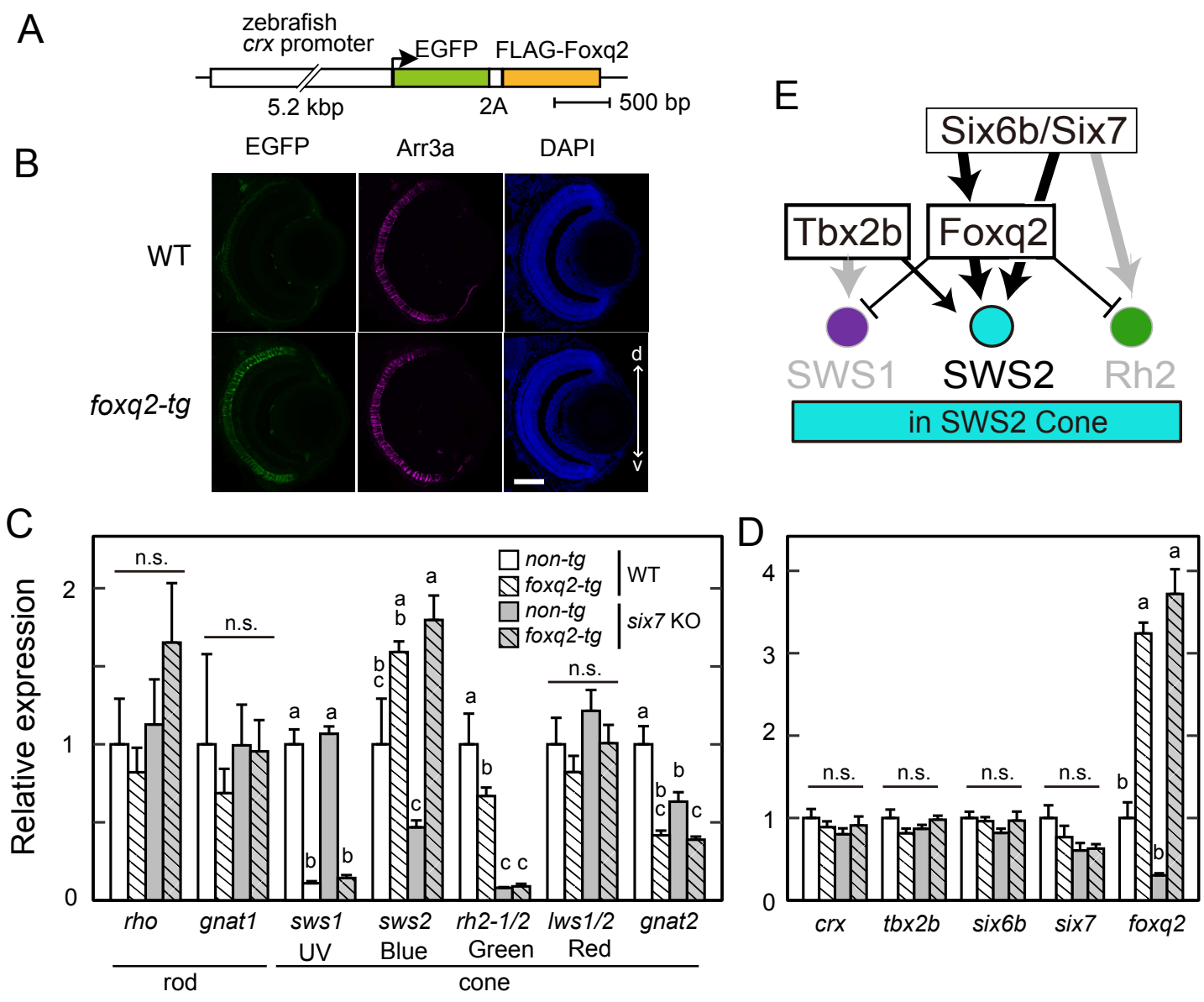

Fig. 4 Foxq2-mediated transcriptional regulation of sws2 downstream of

Six7. (A) Schematic drawing of a transgene construct used for generating foxq2-tg. (B) The immunofluorescent image in the foxq2-tg (ja78Tg) larvae at 5dpf. Scale bar, $50 \mu \mathrm{m}$. d-v, dorsal-ventral retina. (C, D) Relative mRNA levels of opsin genes and transcription factors in the 5-dpf larval eyes of the foxq2-tg (ja78Tg) and/or six7 KO (mean \pm SEM). Distinct letters indicate statistically significant differences ( $P<0.05$ by Tukey's multiple comparison test). n.s., not significant. The number of fish used was as follows: $n=3(\mathrm{WT}), n=5$ ( $\operatorname{six} 7 \mathrm{KO}$ ), $1 n=4$ (foxq2-tg) and $n=5$ (foxq2-tg/six7 KO). (E) Hypothetical model of 2 transcriptional network accounting for sws2 opsin expression in the SWS2 cone 3 subtype. Transcriptional regulation in the SWS2 cone subtype is highlighted in 4 black, while transcriptional regulation in the other cone subtypes is highlighted 5 in grey. 

A

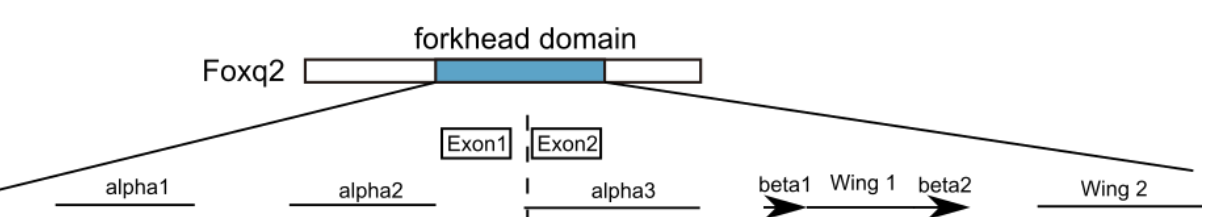

FoxQ2

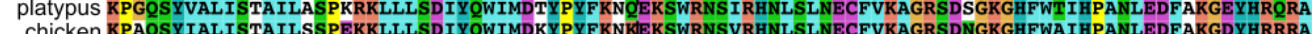
作 SparrowhaWK KPSOSY IALISTAILS PEKKLLLSDI Y QWIMDNY PYFKNKEKSWRNSVRHNLSLNECFVKAGRSDNGKGHFWAIHPANLEDFAKGDYHRRRA
coelacanth KPTQSYIALISMAILS PEKKLLLSDIYQWIMDNYPYFKNKDKSWRNSVRHNLSLNECFIKAGRSDNGKGHFWAIHPANLEDF SKG YHRRRA

ZebrafiSh KPAOSY IALI SMAILDSDEKKLLLCDI YQWIMDHY PYFKS KDKNWRNSVRHNLSLNECF I KAGRSDNGKGHFWAIHPANFQDF SNGDYHRRRA medaka KPNQSY IALISRAILS SKEKKLLLCDI Y WIMDHYPYFKS KDKNWRNSVRHNLSLNECF I KAGRSDNGKGHFWAIHPGNYQDF SKGDYHCRRA Spotted gar KPGQSY IALISTAILASRDKKLLLCDI Y OWIMDNY PYFKSKDKNWRNSVRHNLSLNECFVKAGRSDSGKGHYWA IHPANYQDF SKGD YHRRRA

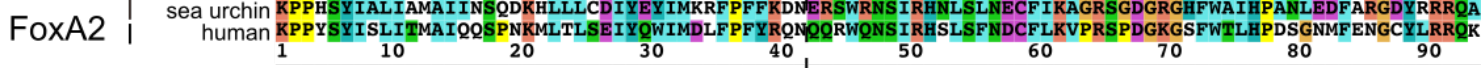

B

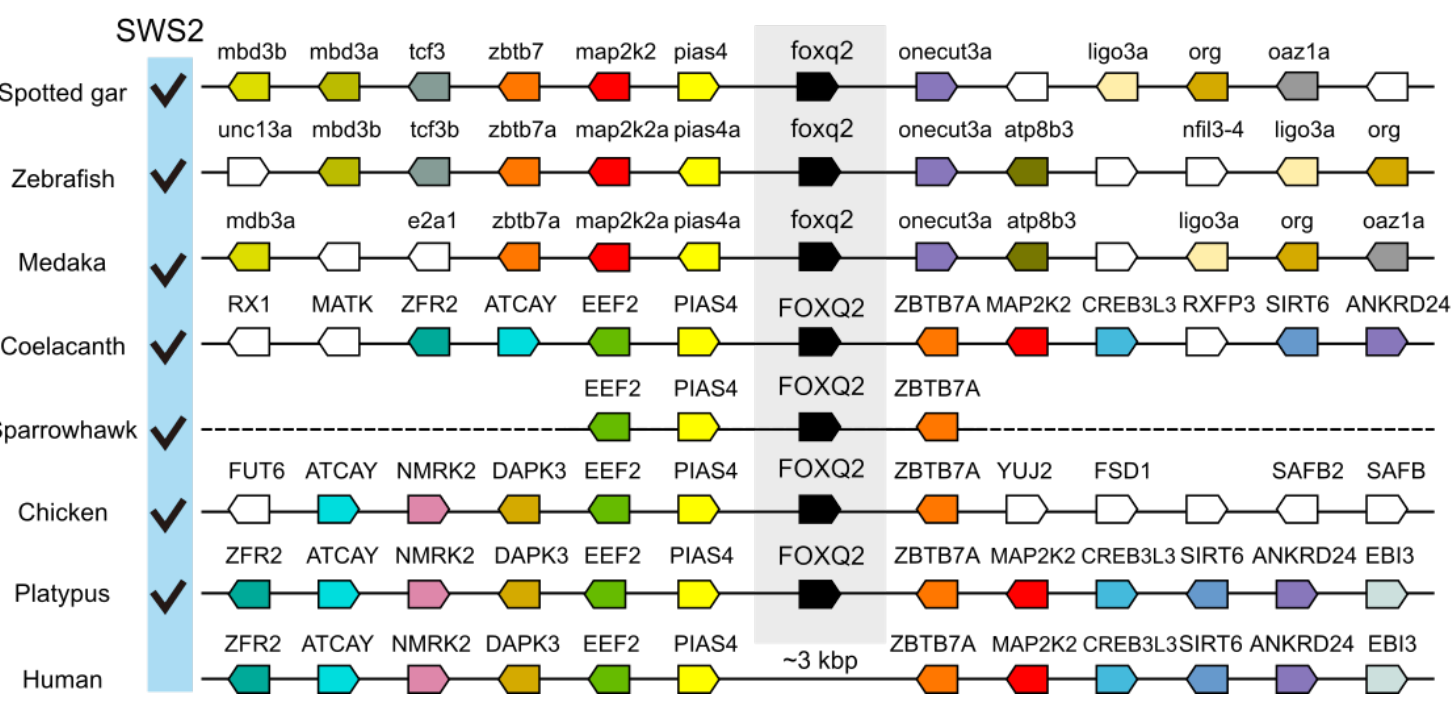

3 Fig. 5. FOXQ2 gene conservation among animal species. (A) Sequence

4 alignment of forkhead domain of FOXQ2 and FOXA2 proteins. Secondary

5 structure elements are indicated at the top according to the FOXA2-DBD/DBE2

6 complex in the previous study (69). (B) Genomic environment of FOXQ2 genes

7 in vertebrate species. Orthologous genes contributing to the conserved order of

8 FOXQ2 genes among vertebrates are similarly color-coded. The presence of

9 SWS2 gene in each species is indicated as checkmarks according to the 10 previous study (70). Accession numbers for the genomic location of FOXQ2 in 11 each species is indicated in Table S5. 